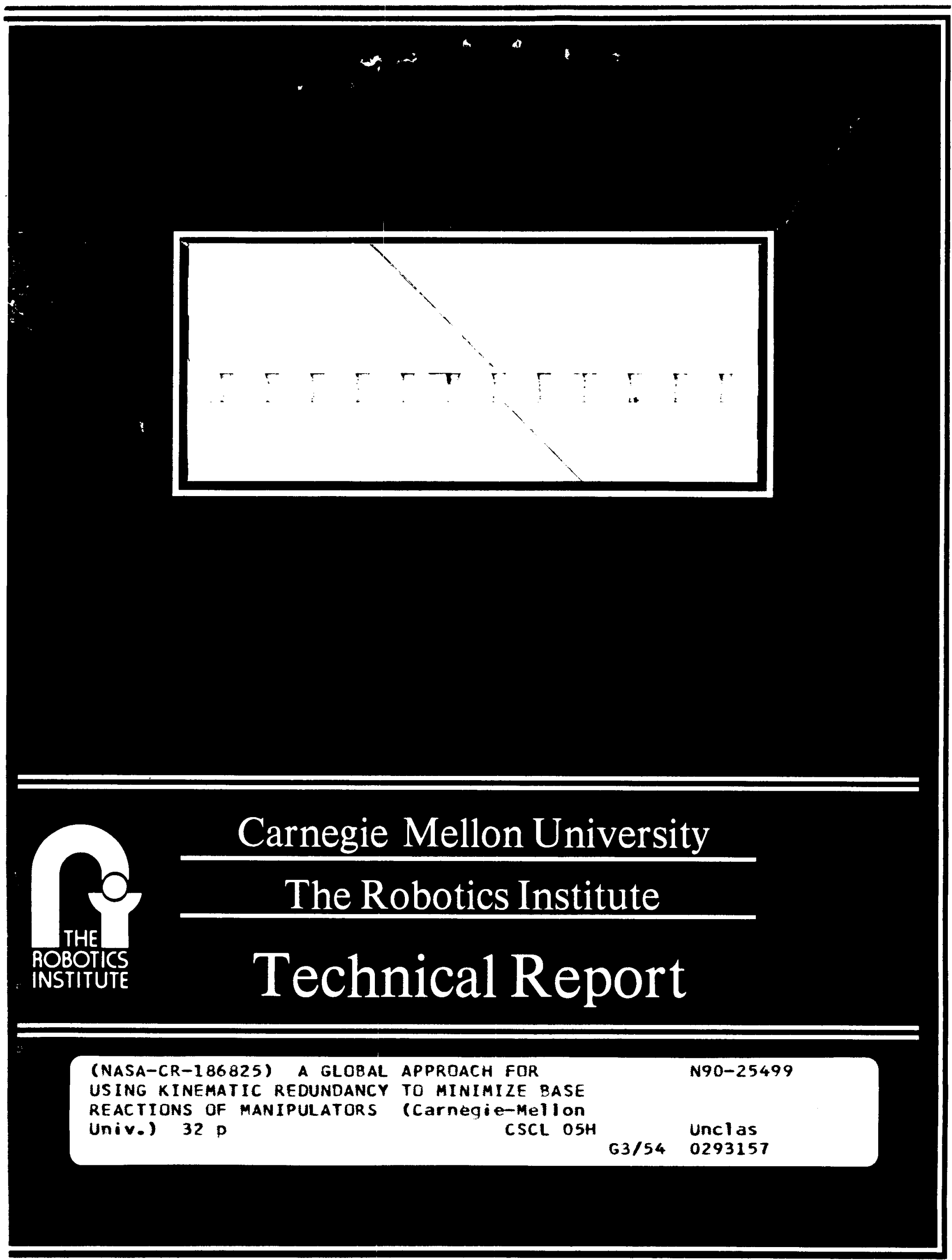

(NASA-CR-186825) A GLOBAL APPROACH FOR

USING KINEMATIC REDUNDANCY TO MINIMIZE BASE

REACTIONS OF MANIPULATORS (Carnegie-MEIION

Univ.) $32 \mathrm{p}$

CSCL O5H

Unclas 


\title{
A Global Approach for Using Kinematic Redundancy to Minimize Base Reactions of Manipulators
}

\author{
C. L. Chung and S. Desa
}

CMU-RI-TR-89-9
Department of Mechanical Engineering
The Robotics Institute
Camegie Mellon University
Pit:tsburgh, Pennsylvania 15213

March 1989

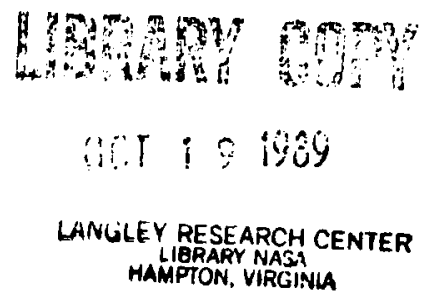

(c) 1989 Carnegie Mellon University

This research has been supported by NASA Lewis Research Center through contract NAG3-811. 


\section{Table of Contents}

1. Introduction

2. Statement of the Problem

3. Description of Approach

3.1 Redundancy Resolution

3.2 Local Approach

3.3 Global Approach

3.3.1 Three-parameter Joint Trajectory Representation

3.3.2 Global Optimization Problem Formulation

3.4 Cost Function

4. Illustrative Example

4.1 End-effector Trajectory

4.2 Cost Function [Case Studies 1-4]

5. Discussions of Results

6. Summary and Conclusions

7. Acknowledgement

Appendixes

Appendix 1 :Equations for 4-3-4 Joint Trajectory Representation

Appendix 2 :Base Reaction Dynamic Equations

Appendix 3 :Physical Dimensions and Mass Properties 


\section{List of Figures}

1.

2.

3.

4.

5.

6.

7.

8.

9.

10.

11.
A $m$ DOF Redundant Manipulator

A Typical 4-3-4 Joint Trajectory Curves Generated by Different Combinations of $\alpha$ and $\beta$ Basic Algorithm for the Global Approach End-effector Trajectory Velocity and Acceleration Profiles

Base Reactions Profiles of Case Study (1)

Base Reactions Profiles of Case Study (2)

Base Reactions Profiles of Case Study (3)

Angular Accelerations Profiles

Manipulator Configurations for the Example

Base Reactions Profiles for Case Study (4) 


\begin{abstract}
An important consideration in the use of manipulators in Microgravity environments is the minimization of the base reactions, i.e. the magnitude of the force and the moment exerted by the manipulator on its base as it performs its tasks. One approach which has been proposed and implemented is to use the redundant degrees of freedom in a kinematically redundant manipulator to plan manipulator trajectories to minimize base reactions. In this paper we develop a global approach for minimizing the magnitude of the base reactions for kinematically redundant manipulators which integrates the Partitioned Jacobian method of redundancy resolution, a 4-3-4 joint-trajectory representation and the minimization of a cost function which is the time-integral of the magnitude of the base reactions. We also compare the global approach with a local approach developed earlier for the case of point-to-point motion of a three degreeof-freedom planar manipulator with one redundant degree-of-freedom. The results of study show that the global approach is more effective in reducing and smoothing the base force while the local approach is superior in reducing the base moment.
\end{abstract}




\section{Introduction}

Recently there has been a lot of interest in the use of kinematically redundant manipulators for various applications like obstacle avoidance and singularity avoidance [2-4]. In this paper we want to utilize the well-known idea that kinematic redundancy provides the analyst or designer with additional degrees of freedom which can be exploited for "useful" purposes. Specifically we are interested in the planning of trajectories for manipulators which are used in space applications [1] and typically operate in microgravity environments.

An issue of considerable importance for manipulators used in Microgravity environments is the minimization of the magnitudes of the dynamic reaction force and moment exerted by the manipulator on its base as it performs its task. One reason for minimizing, and if possible eliminating base reactions, is that the base force and base moment could disturb other tasks or experiments in the vicinity.

In this paper we address the issue of using kinematic redundancy to plan joint-space trajectories which minimize the reactions transmitted by the manipulator to the base. A local approach to this problem is described and implemented in [8-9]: in this approach a performance index or cost function was minimized at each time segment. One of the problems with the local approach was that it led to undesirable "peaks" in the base reactions as shown in Fig.(8). In this paper, we propose and implement a global approach which is based on minimizing an integral performance index over the entire end-effector trajectory.

The rationale which underlies our apjroach is as follows. The trajectory-planning problem for a manipulator reduces to the problem of solving the inverse-kinematic problem for the joint-variables (or "joint-space" trajectory) given the specified trajectory in the task space. In the case of a (kinematically) redundant manipulator the inversekinematic problem has an infinite number of solutions. The basic idea is to pose an optimization problem of minimizing the base reactions in order to obtain joint-space trajectories (which minimize base reactions). In this paper we develop a global approach which integrates the Partitioned Jacobian method of redundant resolution, a 4-3-4 joint-trajectory representation (described in Section 3.3) and the minimization of a cost function which is the time-integral of the magnitude of the base reactions. We also compare the global approach with a local approach developed earlier for the case of point-to-point motion of a three degree-of-freedom planar manipulator with one redundant degree-of-freedom.

The contents of the paper are organized as follows. In Section 2 we develop a statement of the problem to be addressed in this paper. In Section 3, we show how the Partitioned Jacobian method of resolving kinematic redundancy can be used to develop a Global approach for minimizing the magnitude of the base reactions. In this section we also briefly describe the local approach used in $[8,9]$ since we are interested in comparing the two approaches. The global and local approaches are then applied (Section 4) to obtain the optimal joint trajectories which minimize base reactions for a redundant three degrees of freedom planar manipulator (with 1 redundant degree of freedom for its intended task of moving from point-to-point in the workspace). A simple sensitivity study is also carried out in order to get some feel for the "goodness" of the optimized trajectory. The results of the case study (of Section 4) are then discussed in some detail in Section 5. Finally, in Section 6 we summarize the work and draw some conclusions.

\section{Statement of the Problem}

Consider an $m$ degrees of freedom manipulator with $m$ revolute or prismatic joints. Let $q_{i}$ denote the joint variable at joint $i$, the joint variable of each joint is either an angle $\theta_{i}$ (revolute joint) or a distance $d_{i}$ (prismatic joint). For an $m$ degrees of freedom manipulator, the joint displacement vector $q$ can be defined as

$$
\mathbf{q}=\left[\mathrm{q}_{1}, \mathrm{q}_{2}, \ldots, \mathrm{q}_{\mathrm{m}}\right]^{\mathrm{T}} \text {. }
$$

Let $E$ be an arbitrary point on the end-effector as shown in Figure 1. The position of $E$ can be represented with 
reference to the coordinate frame $\{U\}$ fixed in the base by

$$
\mathbf{r}=\left[\begin{array}{llll}
x & y & z
\end{array}\right]^{\mathrm{T}} \text {. }
$$

The kinematic equations relating the end-effector position $\mathbf{r}$ and the joint variable vector $\mathbf{q}$ can be expressed as follows:

$$
\mathbf{r}(t)=\mathbf{g}(\mathbf{q}(t)) \text {. }
$$

where $g$ is a vector of functions which are nonlinear in $q(t)$.

The trajectory planning problem for manipulators is to determine the joint variables $q(t)$ for a specified end-effector trajectory $r(t)$. For a redundant manipulator, the number of degrees of freedom, $m$ is more than the minimum number of degrees of freedom required to perform a task which is denoted by $n$. Therefore, from equation (3) we can see that there are an infinite number of joint space solutions $q(t)$ for a specified end-effector position, $r(t)$. This means that we can take advantage of the redundancy of (kinematically) redundant manipulators to optimize some suitable dynamic criterion. The optimization of this dynamic criterion will then, in general, yield a unique (joint space) solution for the joint variables $q(t)$.

An important problem which arises in the use of manipulators in space is the minimization of the magnitudes of the reaction force and moment transmitted to the base of the manipulator as the end-effector of the manipulator follows a specified trajectory. Let $\mathbf{F}_{\mathrm{o}}$ and $\mathbf{N}_{\mathrm{o}}$, respectively, denote the force vector and moment vector (see Figure 1) transmitted to the base of the manipulator and define

$$
\mathbf{F}_{\mathrm{b}}{ }^{\mathrm{T}}=\left[\mathbf{F}_{\mathrm{o}}^{\mathrm{T}} \mathbf{N}_{\mathrm{o}}^{\mathrm{T}}\right] \text {. }
$$

We will call $F_{b}$ the base reaction vector. The dynamic equations relating the motion of the manipulator to the force and moment transmitted to the base (in a microgravity environment) can be represented in the following form:

$$
F_{b}=M_{b}(\mathbf{q}) \ddot{q}+V(q, \dot{q}),
$$

where $\mathbf{M}_{b}(\mathbf{q})$ is a mass matrix and $\mathbf{V}(\cdot)$ denotes all terms which are nonlinear in $\dot{\mathbf{q}}$. (In Appendix 2 we give expressions for $F_{b}, M_{b}$ and $V$ for a planar 3 d.o.f. manipulator).

Since we are interested in minimizing the base reactions, we must select a cost function B which is an appropriate function of the base reaction vector $F_{b}$, i.e.

$$
B=f\left(\mathbf{F}_{b}\right) \text {. }
$$

Since $F_{b}$ is a function of $(q, \dot{q}, \dot{q}), B$ is also a function of $(q, \dot{q}, \dot{q})$. Therefore minimization of the magnitude of the base forces and base moments reduces to determining the joint space trajectory $q(t)$ which will mimimize the cost function $B$.

We are now in a position to state the objectives of this paper:

1. Given a specified trajectory of the end-effector, use kinematic redundancy to develop an approach for planning the corresponding joint-space trajectories $q(t)$ in order to mimimize a cost function which is a measure of the time-integral of the magnitude of the base reactions. This approach is called the Global Approach.

2. Compare the global approach with the local approach developed in $[8,9]$.

3. Study the sensitivity of the (global) cost function with respect to (small) changes in the joint trajectories.

In the next section we develop the global approach and very briefly describe the local approach. 


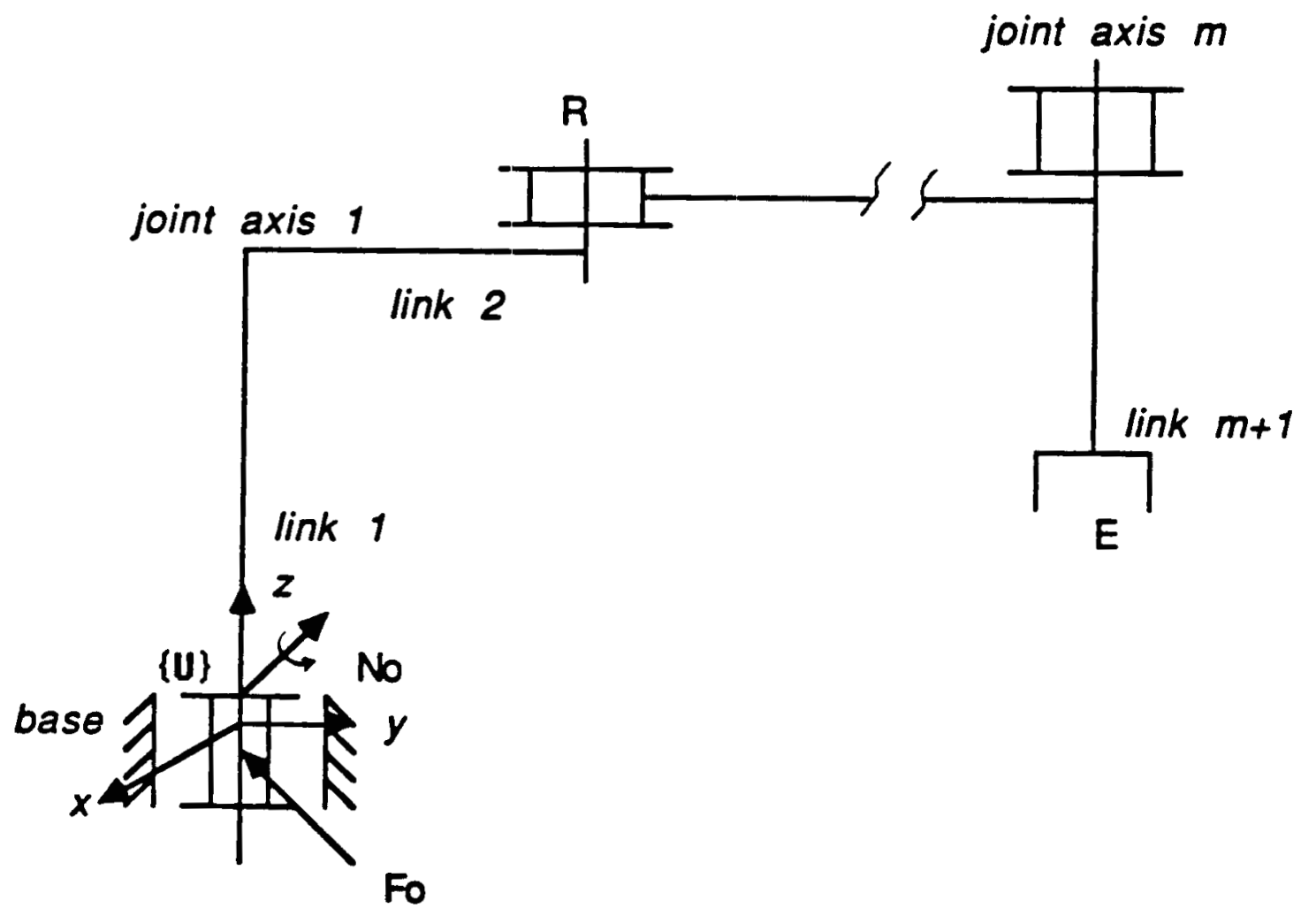

Fig. 1 A, $m$ DOF Redundant Manipulator 


\section{Description of Approach}

The general problem of obtaining unique inverse kinematic solutions for redundant manipulators is termed "redundancy resolution". Even though there are a variety of approaches (which are mostly based on the pseudoinverse of the manipulator Jacobian) proposed for resolving the redundancy of redundant manipulators, relatively few approaches $[5,6,7,8,9]$ address the problem of utilizing redundancy for optimizing dynamic criteria such as torque optimization $[5,6,7]$ or base reaction minimization $[8,9]$. Hollerbach and Suh [5] investigated a few local approaches based on the pseudo-inverse for optimizing joint torques. The results of their study show that the local approach leads to "unstable" solutions for some trajectories. Nakamura [7] proposed a pseudo-inverse approach based on the Pontrayagin's Maximum Principle for globally optimizing the energy used in performing task. Suh and Hollerbach [6] investigated the problem of torque optimization using local and global approaches based on the pseudo-inverse. It was found that the global approach does not have a stability problem and outperforms the local approach [5] at all times. However, the global approach proposed in [6,7] is complex to formulate and solve, and limits on joint angle, joint velocity, and torques cannot be included in the optimization problem. The shortcomings of the pseudo-inverse approach in general are clearly given in [14].

In contrast to the pseudo-inverse approach, we describe an alternative approach, based on partitioning the manipulator Jacobian matrix [4], which is particularly well suited to the base reaction minimization problem. The first application of the Partitioned Jacobian approach to the base reaction minimization problem was a local approach described in $[8,9]$. The primary aim of this paper is to develop and implement a global approach which utilizes the Partitioned Jacobian method of resolving redundancy. The global approach is described in Section 3.3. Since we also want to compare the global approach with the local approach, the local approach is described briefly in Section 3.2. The Partitioned Jacobian method of resolving redundancy is explained in Section 3.1.

\subsection{Redundancy Resolution}

Consider an $m$ degree-of-freedom redundant manipulator. If the velocity $\mathbf{v}$ of a reference point on the end-effector is represented by an $(n \times 1)$ column vector, then we can write the following relationship between $\mathrm{v}$ and the $(m x)$ ) column vector $\dot{\mathbf{q}}$ of rates of change of joint variables:

$$
\mathbf{v}=\mathbf{J}(\mathbf{q}) \dot{q},
$$

where $\mathbf{J}$ is an (nxm) manipulator Jacobian matrix. ( $\mathbf{q}$ will be referred to as the joint "velocity" vector for short.)

Differentiating Eq. (7) with respect to time, the acceleration of the end-effector is

$$
\dot{\mathbf{v}}=\mathbf{j} \dot{\mathbf{q}}+\mathbf{J} \ddot{\mathbf{q}} \text {. }
$$

For redundant manipulators, the Jacobian matrix, $\mathbf{J}$ is not a square matrix. Therefore for a given end-effector velocity $\mathbf{v}$, the Jacobian cannot be inverted to obtain the corresponding joint "velocity" vector $\dot{q}$. In order to obtain joint space solution, we use the Partitioned Jacobian approach developed in [4]. Using this approach, the joint variable vector can be partitioned into,

$$
\mathbf{q}^{T}=\left[\mathbf{q}_{n r}{ }^{T} \mathbf{q}_{r}^{T}\right] \text {, }
$$

where $q_{r^{r}}$ an ( $\left.m-n\right) x l$ column vector, is called the redundant joint vector and $q_{n r}$, an $n \times l$ column vector, is called the nonredundant joint vector.

Eq. (7) can then be written in the form

$$
\mathbf{v}=\mathrm{J}_{n r} \dot{\mathbf{q}}_{n r}+\mathrm{J}_{r} \dot{\mathbf{q}}_{r}
$$


where $\mathrm{J}_{\mathrm{nr}}$ is an $n \times n$ matrix corresponding to $\mathrm{q}_{\mathrm{nr}}$ and $\mathrm{J}_{\mathrm{r}}$ is an $n x(m-n)$ matrix corresponding to $\mathrm{q}_{\mathrm{r}}$.

We have therefore partitioned the Jacobian $\mathbf{J}$ as follows

$$
\mathbf{J}=\left[\begin{array}{ll}
\mathbf{J}_{n r} & \mathbf{J}_{r}
\end{array}\right]
$$

Comment.It is always possible to find a vector of $n$ non-redundant joint variables $q_{n r}$ such that $J_{n r}$ is an invertible (i.e. non- singular) matrix except at. a finite number of singularities.

Eq. (8) can be written as follows:

$$
\dot{\mathbf{v}}=\dot{\mathrm{J}}_{n r} \dot{\mathbf{q}}_{n r}+\dot{\mathrm{j}}_{r} \dot{\mathbf{q}}_{r}+\mathrm{J}_{n r} \ddot{\mathbf{q}}_{n r}+\mathrm{J}_{r} \ddot{\mathbf{q}}_{r}
$$

Rearranging Eqs. (10) and (12), we have

$$
\begin{aligned}
& \dot{\mathbf{q}}_{n r}=\mathbf{J}_{n r}{ }^{-1}\left(\mathbf{v}-\mathbf{J}_{r} \dot{\mathbf{q}}_{r}\right) \\
& \ddot{\mathbf{q}}_{n r}=\mathbf{J}_{n r}{ }^{-1}\left(\dot{\mathrm{v}}-\dot{J}_{r} \dot{\mathbf{q}}_{r}-\dot{\mathrm{J}}_{n r} \dot{\mathrm{q}}_{n r}-\mathrm{J}_{r} \ddot{\mathbf{q}}_{r}\right) .
\end{aligned}
$$

In the approaches described below, one specifies the redundant joint variable vector $q_{r}(t)$ to be a convenient (vector) function of time from which $\dot{\mathrm{q}}_{\mathrm{r}}(t)$ is readily obtained. Equation (13) can then be used to determine the non-redundant joint variable velocity vector $\dot{q}_{n r}(t)$; from the latter $q_{n r}(t)$ can be obtained by integration.

\subsection{Local Approach}

In the local approach, the total time of the task is divided into a number of time segments. For convenience, assume that the duration for each time segment is the same. If $\dot{q}_{\mathrm{po}}$ is the joint velocity vector of the redundant joints at the start of any time-segment $\Delta t$ and $\dot{q}_{\mathrm{r}}$ is the joint velocity vector of the redundant joints at the end of the time segment, then we can write

$$
\dot{\mathbf{q}}_{r}=\dot{\mathbf{q}}_{r o}+\Delta \dot{q}_{r},
$$

where $\Delta \dot{\mathrm{q}}_{\mathrm{r}}$ can be approximated by a linear combination of shape-function $\mathrm{f}^{\mathrm{i}}(\mathrm{t}), \mathrm{i}=1,2, \ldots, \mathrm{k}$ as follows

$$
\begin{aligned}
& \Delta \dot{q}_{r}^{1}=c_{11} f^{1}(t)+c_{12} f^{2}(t)+\ldots+c_{1 k} f^{k}(t) \\
& \Delta \dot{q}_{r}^{2}=c_{21} f^{\prime}(t)+c_{22} f^{2}(t)+\ldots+c_{2 k} f^{*}(t) \\
& \Delta \dot{q}_{r}^{(m-n)}=c_{(m-n) 1} f^{1}(t)+c_{(m-n) 2} f^{2}(t)+\ldots+c_{(m-n) k} f^{k}(t),
\end{aligned}
$$

where $\Delta \dot{q}_{r}, i=(1,2, \ldots .,(m-n))$, is the $\imath^{\text {th }}$ element of $\Delta \dot{q}_{r}$. The use of shape functions in optimization of torques of non-redundant manipulators is described in [13].

If $\mathrm{C}$ denotes the matrix of parameters $c_{\mathrm{ij}}, \mathrm{i}=(1, \ldots,(\mathrm{m}-\mathrm{n})), \mathrm{j}=(1,2, \ldots \mathrm{k})$, and $\mathrm{f}(\mathrm{t})$ is a column vector whose $i^{i \text { h }}$ element is $f_{i}(t)$, then Eq. (16) can be written in a more compact form

$$
\Delta \dot{q}_{r}=\operatorname{Cr}(t) \text {. }
$$

Combining Eq.(15) and Eq.(17) we obtain 


$$
\dot{\mathbf{q}}_{r}=\dot{\mathbf{q}}_{r o}+\mathrm{Cr}(t) \text {. }
$$

For convenience, $\mathrm{f}^{\mathrm{i}}(\mathrm{t})$ is chosen to be polynomials $\mathrm{t}^{\mathrm{i}-1},(\mathrm{i}=1,2, \ldots, \mathrm{k})$ and therefore $\mathrm{f}(\mathrm{t})$ is given by

$$
f(t)=\left[\begin{array}{lllllll}
1 & t & t^{2} & \ldots & t^{k-1}
\end{array}\right]^{T} \text {, }
$$

where $k$ is the degree of the polynomial selected for $q_{r}$.

Substituting Eq.(18) in Eqs.(13-14), they become

$$
\begin{aligned}
& \dot{\mathrm{q}}_{\mathrm{nr}}=\mathrm{J}_{\mathrm{nr}}^{-1}\left[\mathrm{v}-\mathrm{J}_{\mathrm{r}}\left(\dot{\mathrm{q}}_{\mathrm{ro}}+\mathrm{C}\right)\right] \\
& \ddot{\mathrm{q}}_{\mathrm{nr}}=\mathrm{J}_{\mathrm{nr}}^{-1}\left[\dot{\mathrm{v}}-\dot{\mathrm{J}}_{\mathrm{r}}\left(\dot{\mathrm{q}}_{\mathrm{ro}}+\mathrm{Cr}\right)-\mathrm{J}_{\mathrm{nr}} \mathrm{J}_{\mathrm{nr}}^{-1}\left[\mathrm{v}-\mathrm{J}_{\mathrm{r}}\left(\dot{\mathrm{q}}_{\mathrm{ro}}+\mathrm{Cr}\right)\right]-\mathrm{J}_{\mathrm{r}} \mathrm{C} \dot{f}\right] .
\end{aligned}
$$

Now, we can compute the values of $\mathbf{q}, \dot{\mathbf{q}}$, and $\ddot{\mathbf{q}}$ as functions of the elements $c_{i j}$ of the $\mathbf{C}$ matrix for each time segment. For an arbitrary manipulator configuration, the base reactions $\left(F_{0}\right.$ and $\left.N_{0}\right)$ at the end of each time step are therefore functions of the elements of the $C$ matrix. In Section 3.4 (see Eq. 37) we will present a local cost function, $\mathrm{B}(\mathrm{C})$ that is a measure of the magnitudes of the base force and base moment at the end of each time segment. Using that cost function we can pose the inverse kinematic problem as an minimization problem which yields the optimal C matrix for each time segment. For further details refer to $[8,9]$.

\subsection{Global Approach}

In contrast to the local approach where the magnitudes of the base reactions at the end of each time segment is optimized, the global approach described below optimizes a cost function which is a time-integral of the magnitudes of the base force and base moment over the whole time interval, $\tau_{\mathrm{f}}$.

Using the redundancy resolution scheme developed in Section 3.1, the redundant joint trajectories are used in the optimization of appropriate dynamic criteria such as the minimization of the magnitudes of the base reactions. The redundant joint velocity vector representation, Eq. (18) used in the local approach would be difficult to implement for a time integral cost function. In the local approach, for each time segment we have $(m-n) \times k$ parameters (i.e. the elements $c_{i j}$ of the matrix $C$ ). If the total time is divided into $l$ time segments, then we would have $l x(m-n) x k$ parameters to determine for global approach. To keep the number of parameters required for the representation of the redundant joint variables small, we use the simpler representation described below.

One of the intuitive requirements for the redundant joint trajectories is that the joint trajectories have to be smooth. Also, the angular velocity and acceleration at both of the initial and final points of the redundant joint trajectories should be zero. One way of representing this family of curves is the use of higher order polynomials (splines) to satisfy the velocity and accelerations boundary conditions. Since very high order polynomials exhibit unpredicted behaviors, a popular method is to break a joint trajectory into a number of segments which can be represented by lower order polynomials such as third and fourth order ones. These segments will satisfy the boundary conditions and are continuous from one segment to another. The representation considered in this paper is a three-segment method which consists of two fourth-order polynomials for the initial and final segments and a third order polynomial for the transition segment. This method is known as the 4-3-4 joint trajectory representation. The details of this representation can be found in [10]. As shown in Figure 2, a typical 4-3-4 joint trajectory for each joint consists of three segments:an initial section $\left(\theta_{1}(t)\right)$, a transition section $\left(\theta_{2}(t)\right)$, and a final section $\left(\theta_{3}(t)\right)$. Basically, curves described by this method are functions of the initial and final joint points $\left(\theta_{i}\right.$ and $\left.\theta_{f}\right)$, the time period of the transition segment $\left(\tau_{2}\right)$, the total time $\left(\tau_{\mathrm{f}}\right)$ and the angular displacement traversed during the transition period $\left(\delta_{2}\right)$. The expressions for $\theta_{1}(t), \theta_{2}(t)$, and $\theta_{3}(t)$ are given in Appendix 1 . 


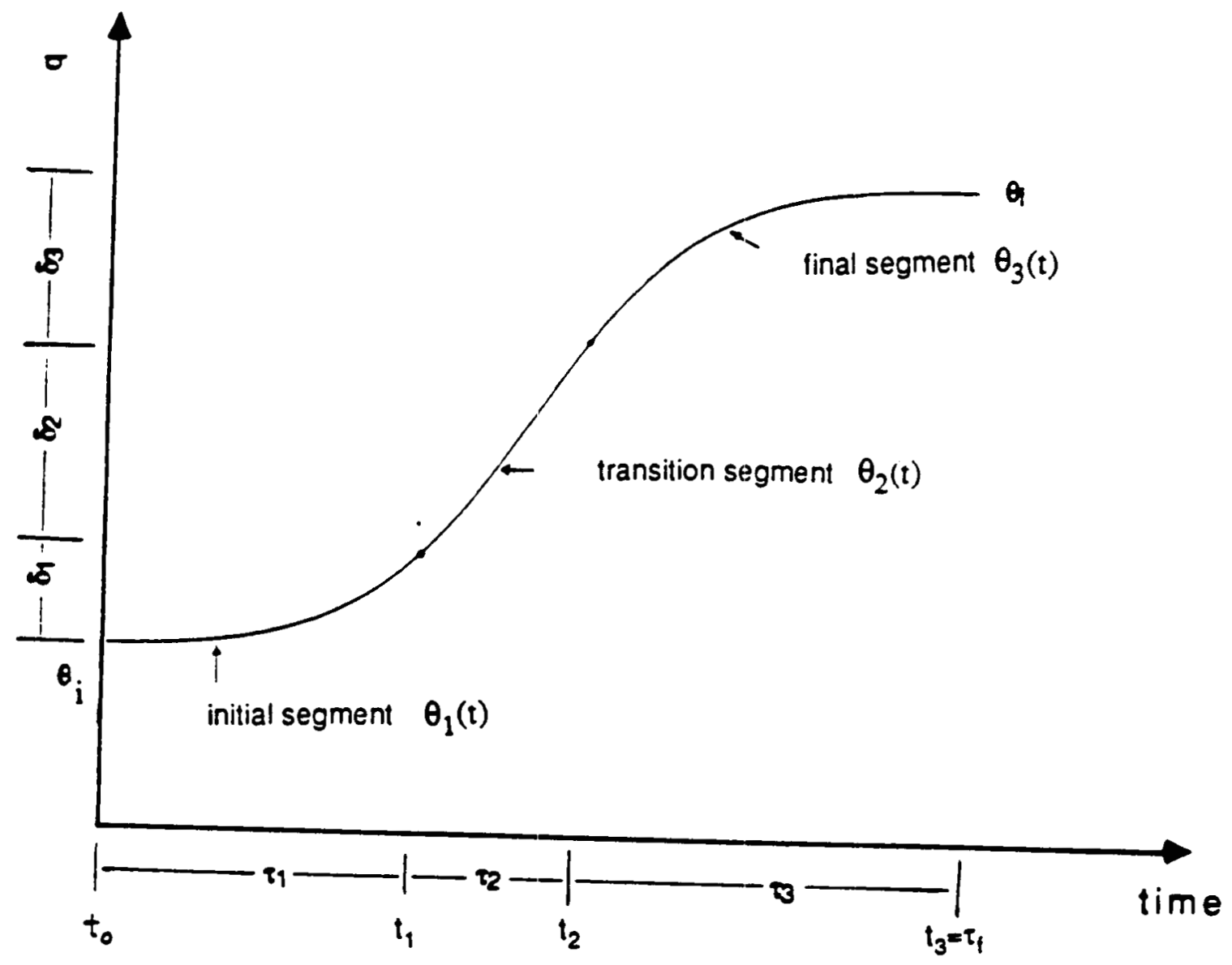

Fig. 2 A Typical 4-3.4 Joint Trajectory 


\subsubsection{Three-parameter Joint Trajectory Representation}

The 4-3-4 joint trajectory can be simplified by considering the following normalized parameters: (a) time factor, $\beta(0<\beta<1)$

$$
\beta=\frac{\tau_{2}}{\tau_{1}+\tau_{2}+\tau_{3}},
$$

where $\tau_{i}$ 's are time intervals for the three segments (see Fig. 2);

(b) angular displacement factor, $\alpha$

$$
\alpha=\frac{\delta_{2}}{\delta_{1}+\delta_{2}+\delta_{3}},
$$

where $\delta_{i}$ 's are angular displacements traversed during the $i$ th time interval (see Fig. 2), $i=1,2,3$.

For $\tau_{1}=\tau_{3}$ and $\delta_{1}=\delta_{3}$, we have the following relationships:

$$
\begin{aligned}
& \delta_{1}=\theta_{f o} \alpha^{\prime} \\
& \delta_{2}=\theta_{f o} \alpha \\
& \delta_{3}=\theta_{f o} \alpha^{\prime} \\
& \tau_{1}=\tau_{f} \beta^{\prime} \\
& \tau_{2}=\tau_{f} \beta \\
& \tau_{3}=\tau_{f} \beta^{\prime},
\end{aligned}
$$

where $\theta_{\mathrm{fo}}=\theta_{\mathrm{f}}-\theta_{\mathrm{i}}, \tau_{\mathrm{f}}$ is the total time of the task, $\alpha^{\prime}=(1-\alpha) / 2$ and $\beta^{\prime}=(1-\beta) / 2$.

Note: the above variables, defined by Eqs. (24) through (29) are functions of $\alpha, \tau_{f}, \beta, \theta_{i}$, and $\theta_{f}$. In general, for the redundant manipulator inverse kinematic problem, the initial angular position $\left(\theta_{i}\right)$ for the redundant joint variable and the total time of the task $\left(\tau_{f}\right)$ are usually specified. Therefore, there are only three unknown paremeters, namely $\alpha, \theta_{\mathrm{f}}$, and $\beta$ for each degree of redundancy. Using these parameters, we obtain a three-parameter joint trajectory representation (based on the 4-3-4 joint trajectory representation) for the redundant joint variables. The $j^{\text {th }}$ element of the redundant joint velocity vector, $\mathrm{q}_{\mathrm{r}}^{\mathrm{j}}$ can be expressed as

$$
q_{r}^{j}(t)=\theta_{k}^{j}(t) \text { for } t_{k-1} \leq k t_{k}, k=1,2,3
$$

where $\theta_{k}^{j}(t)$ denotes the polynomial for the $k^{\text {th }}$ segment, $(k=1,2,3)$, of the $f^{\text {th }}$ redundant joint variable, $j=1,2, . .(m-n)$ and $t_{k}$ 's are shown in Fig. 2.

Figure 3 illustrates the curves generated by different combinations of $\alpha$ and $\beta$. Note that these curves are smooth and satisfy the boundary conditions.

In the next section, we show how the three-parameter joint trajectory representation for redundant joint variables can be used to find a joint space solution (i.e. inverse kinematic solution) for the redundant manipulators.

\subsubsection{Global Optimization Problem Formulation}

For a redundant manipulator with $(m-n)$ degrees of redundancy, the application of the three-parameter scheme to represent each of the $(m-n)$ redundant joint variables yields $\mathrm{q}_{\mathrm{r}}$ as a function of $3 x(m-n)$ number of parameters, $\alpha^{j}$, $\beta j$, and $\theta_{f}^{j}, j=1,2, . .(m-n)$, which need to be determined. Using Eq. (13), $q_{n r}$ can also be determined as a function of these $3 x(m-n)$ parameters. Let $\underline{\alpha}, \underline{\beta}$, and $\underline{\theta}_{f}$ denote $(m-n) x l$ column vectors whose $j^{\text {th }}$ element is denoted, respectively, by $\alpha^{j}, \beta^{j}$, and $\theta_{\mathrm{f}}^{j}$. 
The cost function B which is a function of the base reactions and therefore a function of $q, \dot{q}, \ddot{q}$, can be expressed as a function of $\underline{\alpha}, \underline{\beta}$, and $\underline{\theta}_{f}$ (see procedure below for details). Therefore the optimization problem can be posed as follows:

$$
\operatorname{minimize} B\left(\underline{\alpha}, \underline{\beta}, \underline{\theta}_{f}\right)
$$

subject to the constraints

$$
\begin{aligned}
& \theta_{\min }^{j}<\theta_{\mathrm{f}}^{j}<\theta_{\max }^{j} \\
& 0<\alpha^{j}<1, \text { and } \\
& 0<\beta^{j}<1, j=1,2, . .(m-n) .
\end{aligned}
$$

For the global approach B is a time-integral cost function defined in Section 3.4 (Eq. 35) below, which is a measure of the magnitude of the base reactions. $\theta_{\min }$ and $\theta_{\max }^{j}$ are selected based on the information on the joint limits.

Fig. 4 depicts a simplified flow-chart which captures the essence of the the global approach. The procedure for the global approach, corresponding to the flowchart in Fig. 4, is as follows:

1. Initialize the parameters vectors $\underline{\alpha}, \underline{\beta}$, and $\underline{\theta}_{f}$.

2. Using the 4-3-4 redundant joint representation (Eq. (30) and Appendix 1) obtain $\mathbf{q}_{\mathrm{r}}, \dot{\mathrm{q}}_{\mathrm{r}}$, and $\ddot{\mathbf{q}}_{\mathrm{r}}$ as functions of $\underline{\alpha}, \underline{\beta}$, and $\underline{\theta}_{f}$ for the time interval $\tau_{f}$.

3. Using Eq. (13) and (14), respectively, to determine $\dot{\mathbf{q}}_{\mathrm{nr}}$ and $\ddot{\mathrm{q}}_{\mathrm{nr}}$, as a function of $\underline{\alpha}, \underline{\beta}$, and $\underline{\theta}_{\text {f. }}$.

4. Obtain the non-redundant joint variable vector $q_{n r}$ by integrating $\dot{q}_{n r}$ (obtained in Step 3). We now have $q, \dot{q}$, and $\ddot{q}$ as a function of $\underline{\alpha}, \underline{\beta}$, and $\underline{\theta}_{f}$.

5. Use the dynamic equations (Eq. (5)) and the results of Step 4 to determine the base reaction vector $F_{b}$, as a function of $\underline{\alpha}, \underline{\beta}$, and $\underline{\theta}_{f}$.

6. Define a suitable time-integral cost function $B$ of the base reactions $F_{b}$ (see Sections 3.4 and 4.2).

7. Combine the results of steps 5 and 6 to obtain the cost function B as a function of $\underline{\alpha}, \underline{\beta}$, and $\underline{\theta}_{f}$.

8. Apply the Hooke and Jeeves optimization method [12], to update the parameter vectors $\underline{\alpha}, \underline{\beta}$, and $\underline{\theta}_{f}$.

9. Repeat Steps 2 through 8 until the parameters vectors converge to their optimal values $\underline{\hat{\alpha}}, \hat{\underline{\beta}}, \hat{\theta}_{\text {f }}$. Once the optimal values of $\underline{\alpha}, \underline{\beta}$, and $\underline{\theta}_{f}$ have been obtained, the optimal joint space trajectories vector $q(t)$ are readily determined.

\subsection{Cost Function}

The selection of a suitable cost function is crucial in obtaining a joint space solution that minimizes base reactions. In this section, we discuss the issue of selecting a proper cost function which can be used as a measure of the magnitudes of the base reactions $\left(\mathbf{F}_{0}\right.$ and $\left.\mathbf{N}_{0}\right)$.

In general, the magnitude of the base force is given by

$$
F_{0}=\left(F_{o 1}{ }^{2}+F_{02}{ }^{2}+F_{03}{ }^{2}\right)^{1 / 2}
$$

and the magnitude of the base moment is

$$
\mathrm{N}_{0}=\left(\mathrm{N}_{01}^{2}+\mathrm{N}_{02}{ }^{2}+\mathrm{N}_{03}{ }^{2}\right)^{1 / 2}
$$

We introduce two weighting factors $\left(w_{1}\right.$ and $w_{2}$ ) that enable us to place appropriate weights on the base force 


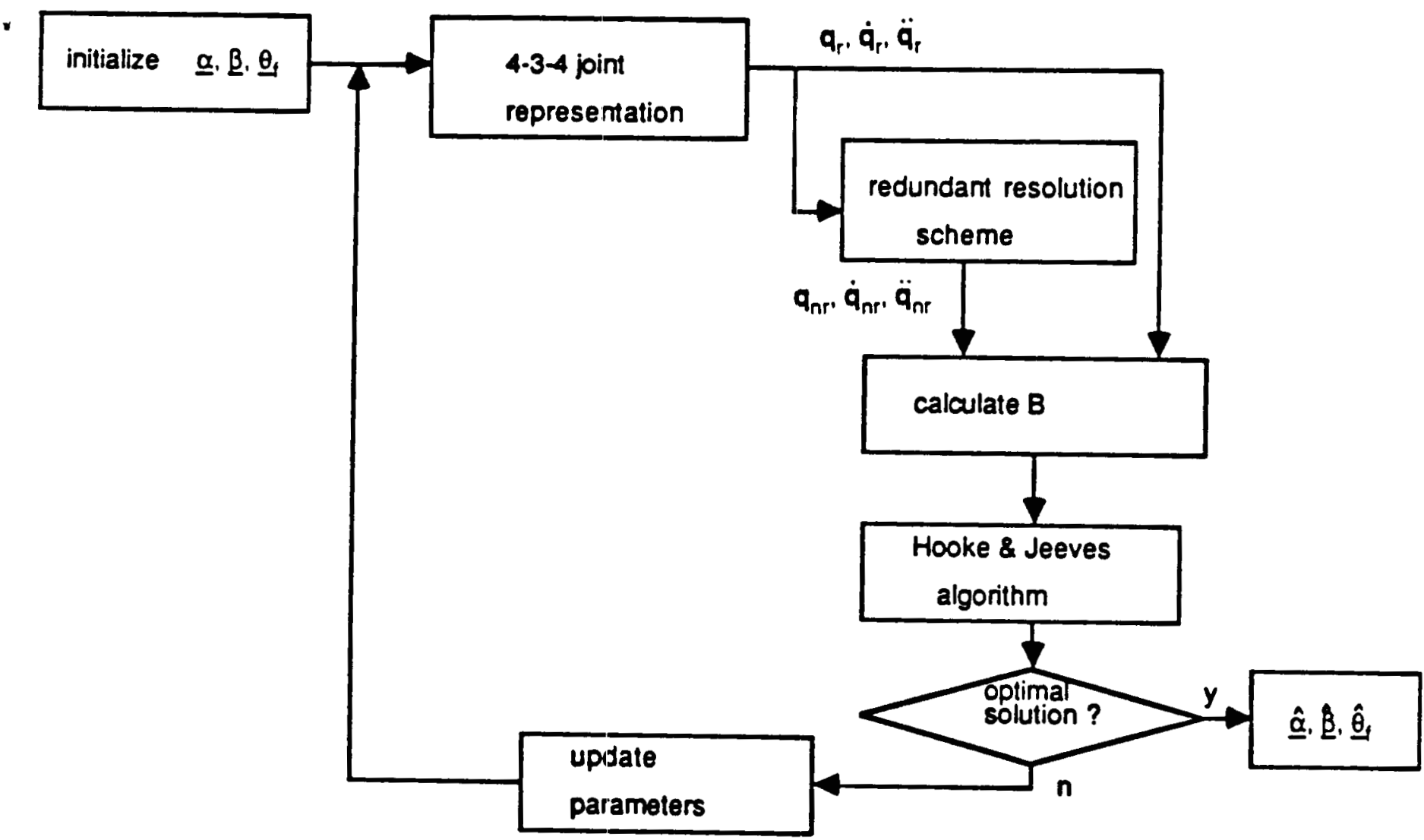

Fig. 4 Basic Algorithm For the Global Approach 
and base moment components. The cost function $B$ can be expressed as

$$
\begin{aligned}
& B=w_{1} F_{o}^{2}+w_{2} N_{o}^{2}, \text { for the local approach, and } \\
& B=\int_{0}^{\tau} w_{1} F_{0}^{2}+w_{2} N_{o}^{2} d t, \text { for the global approach. }
\end{aligned}
$$

Define

$$
Q=\left[\begin{array}{ll}
w_{1} I & 0 \\
0 & w_{2} I
\end{array}\right]
$$

We can write Egs. (34) and (35) in a more compact matrix form as follows:

$$
\mathbf{B}=\mathbf{F}_{\mathrm{b}}^{\mathrm{T}} \mathbf{Q} \mathbf{F}_{\mathrm{b}}
$$

and

$$
B=\int_{0}^{\tau} \mathbf{F}_{b}^{T} \mathbf{Q} \mathbf{F}_{b} d t
$$

The weights $w_{1}$ and $w_{2}$ must be carefully chosen to : (1)appropriately scale the base reactions with respect to a reference set of base reactions and (2)ensure that all quantities in the cost function are dimensionally homogeneous. The choice of appropriate weights will be dealt with in Section 4.2.

\section{Illustrative Example}

In this section we apply the basic approaches described in the paper to minimizing the base reactions of a three degree-of-freedom planar manipulator. The task is a point-to-point motion of the end-effector from a specified initial position to a specified final position. Using the above approaches, we would like to plan a trajectory in the joint space to minimize the magnitudes of the base reactions as the manipulator moves from the initial position to the final position.

In this problem, for the planar manipulator, $m=3$; since we are interested in the position of the end-effector, $n=2$. Therefore we have $(m-n)=1$ degree of redundancy. The redundant link of this problem is chosen to be the first link which is attached to the base $\left(q_{r}=q_{1}\right)$.

The base reactions dynamic equations of the 3 d.o.f. planar manipulator are in Appendix 2 and the link dimensions and mass properties are given in Appendix 3.

We sudy the following four cases:

1. The minimization of the time-integral of the magnitude of the base moment only.

2. The minimization of the time-integral of the magnitude of the base force only.

3. The minimization of the time-integral of an appropriately weighted combination of the magnitudes of the base force and base moment. We use the results of cases (1) and (2) to show how the weights $w_{1}$ and $w_{2}$ should be chosen.

4. A sensitivity analysis of the sensitivity of the cost function of Case (3) with respect to small changes in (the parameters of) the joint space trajectories. 


\subsection{End-effector Trajectory}

The distance measured from the initial position along a straight line which connects the initial position and the final position can be expressed as

$$
d(t)=b\left(t-\frac{\tau_{f}}{2 \pi} \sin \left(\frac{2 \pi t}{\tau_{f}}\right)\right)
$$

where $b$ is an arbitrary constant and $\tau_{f}$ is the total time of the task. The speed of the end effector is given by

$$
v(t)=b\left(1-\cos \left(\frac{2 \pi l}{\tau_{f}}\right)\right) \text {. }
$$

Fig. 5 shows the desired velocity and acceleration profiles of the end-effector.

The initial position of the end effector is $(x, y)=(0.0,1.2 .071)(\mathrm{m})$ which corresponds to an initial manipulator configuration, $q^{T}=\left[45^{\circ}, 45^{\circ}, 45^{\circ}\right]$. The desired final position is $(x, y)=(0.3536,0.8535)(\mathrm{m})$. The following values are chosen for the trajectory:

$\tau_{\mathrm{f}}=2.0 \mathrm{~s}$

$\mathrm{b}=0.25$

$\mathrm{d}\left(\tau_{\mathrm{f}}\right)=0.5 \mathrm{~m}$

$\Delta \mathrm{t}=0.1 \mathrm{~s}$.

\subsection{Cost function [Case Studies 1 - 4]}

To minimize the base reactions properly, it is important to select a suitable weighting matrix $Q$ for the cost function. Therefore, the choices of the two weighting factors $\left(w_{1}\right.$ and $\left.w_{2}\right)$ are crucial. To understand the effects of these weighting factors on the base reactions, three cases of different $Q$ matrices are studied. We only show the cost functions for the global approach. The cost function for the local approach can be obtained by Eq. (37).

\section{(a)Case Study 1}

In this case study we select a cost function that only minimizes the magnitude of the base moment in order to determine how small the magnitude of the base moment can be made. We simply choose $w_{I}=0$ and $w_{2}=1$ in Eq. (36) to obtain the weighting matrix

$$
Q_{1}=\left[\begin{array}{ll}
0 & 0 \\
0 & I
\end{array}\right] \text {. }
$$

and the corresponding cost function (from Eq. 38)

$$
B=\int_{0}^{\tau} N_{0}^{2} d t
$$

\section{(b) Case Study 2}

In this case, we want to know the extent to which the magnitude of the base force component can be minimized by choosing $w_{1}=1$ and $w_{2}=0$ in Eq. (36) to obtain the matrix

$$
\mathbf{Q}_{2}=\left[\begin{array}{ll}
1 & 0 \\
0 & 0
\end{array}\right] \text {, }
$$

and the corresponding cost function (from Eq. 38) takes the form

$$
B=\int_{0}^{\tau} F_{0}^{2} d t
$$




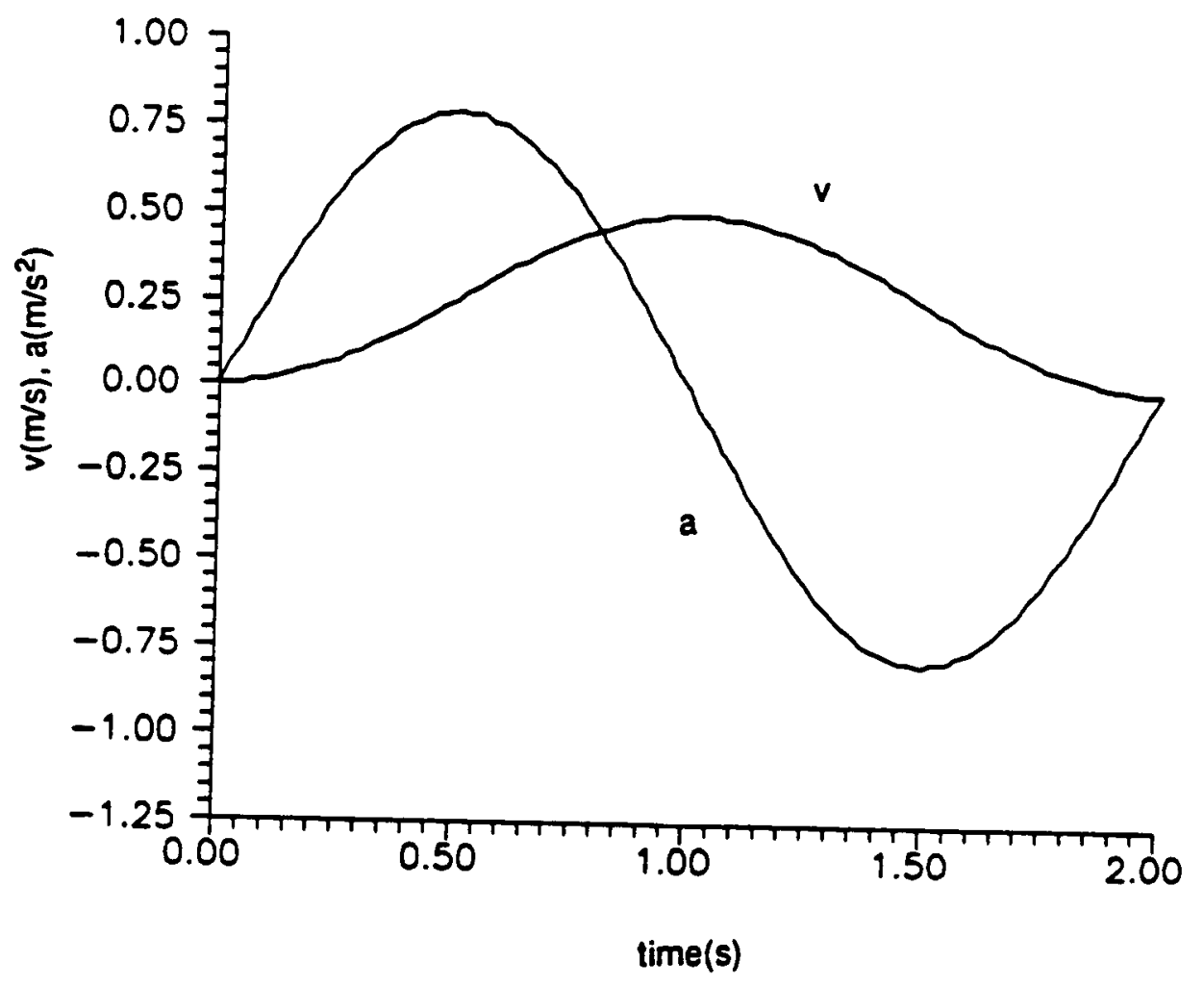

Fig. 5 End-effector Trajectory Velocity and Acceleration Profiles 
(c) Case Study 3

In general, we want to minimize the magnitudes of both the base force, $F_{0}$ and the base moment, $N_{0}$ transmitted to the base. Since $F_{0}$ and $N_{0}$ are different physical quantities, we require a weighting matrix $Q$ to non-dimensionalize and to appropriately scale the base force and base moment components. The results of Case studies 1 and 2 are important in this regard.

The average value of the base force $\left(F_{0}\right)$ transmitted to the base in Case Study $1, F_{\text {avg }}$ is a measure of how small we can make the base force. Similarly, the average value of the moment transmitted to the base in Case Study $2, \mathrm{~N}_{\mathrm{avg}}$ is a measure of how small we can make the magnitude of the base moment. We therefore choose,

$$
B=\int_{0}^{\tau} f\left(\frac{f_{o}^{2}}{f_{\text {avg }}^{2}}+\frac{N_{o}^{2}}{N_{\text {avg }}^{2}}\right) d t .
$$

The weighting matrix therefore is given by

where

$$
Q_{3}=\left[\begin{array}{ll}
w_{1} I & 0 \\
0 & w_{2} I
\end{array}\right] \text {, }
$$

$$
\begin{aligned}
& w_{1}=\frac{1}{F_{\text {avg }}^{2}} \\
& w_{2}=\frac{1}{N_{\text {avg }}^{2}} .
\end{aligned}
$$

Note that the above choices of $w_{1}$ and $w_{2}$ simultaneously achieve the desired scaling and nondimensionalization.

\section{(d)Case Study 4 (Sensitivity)}

In practice, it is impossible to realize the exact optimal joint trajectories due to the errors in the control system and noise and uncertainties in the system. Therefore, it is important to examine how sensitive the base reactions are to the deviations of the joint trajectories from the optimal solution. The global approach provides us a simple means of studying this issue. One way of studying this issue is to introduce perturbations about the optimal values of $\alpha, \beta$, and $\theta_{\mathrm{f}}$ obtained from Case Study 3. The following three cases in each of which one of the three parameters is increased by $20 \%$ were studied:

case a : the optimal value of $\alpha$, viz $\hat{\alpha}$ is replaced by $1.2 \hat{\alpha}$

case $b$ : the optimal value of $\beta$, viz $\hat{\beta}$ is replaced by $1.2 \hat{\beta}$

case $c$ : the optimal value of $\theta_{f}$, viz $\hat{\theta}_{f}$ is replaced by $1.2 \hat{\theta}_{f}$.

In the next section, the results of these case studies will be presented and discussed.

\section{Discussions of Results}

We are interested in the effectiveness of the proposed global approach in minimizing the magnitudes of the base force and base moment as well as in comparing this approach with the local approach. We therefore, plotted the magnitudes of the base force and base moment (corresponding to the optimized trajectory) for each of three cases enumerated in the previous section. From an examination of the results shown in Figs. 6 through 11, the following imporant conclusions/points should be noted: 


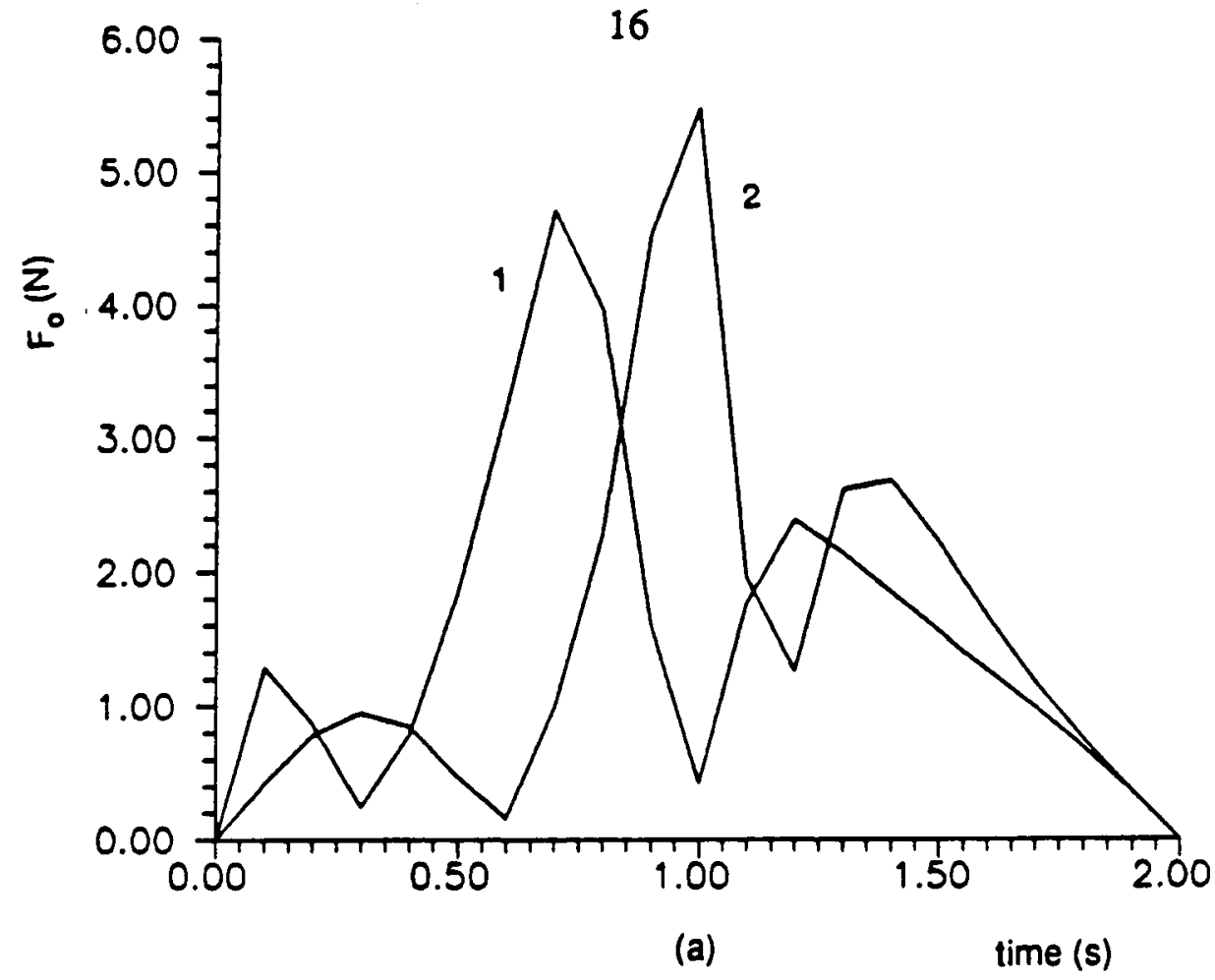

$1 \cdot$ local

2 - global

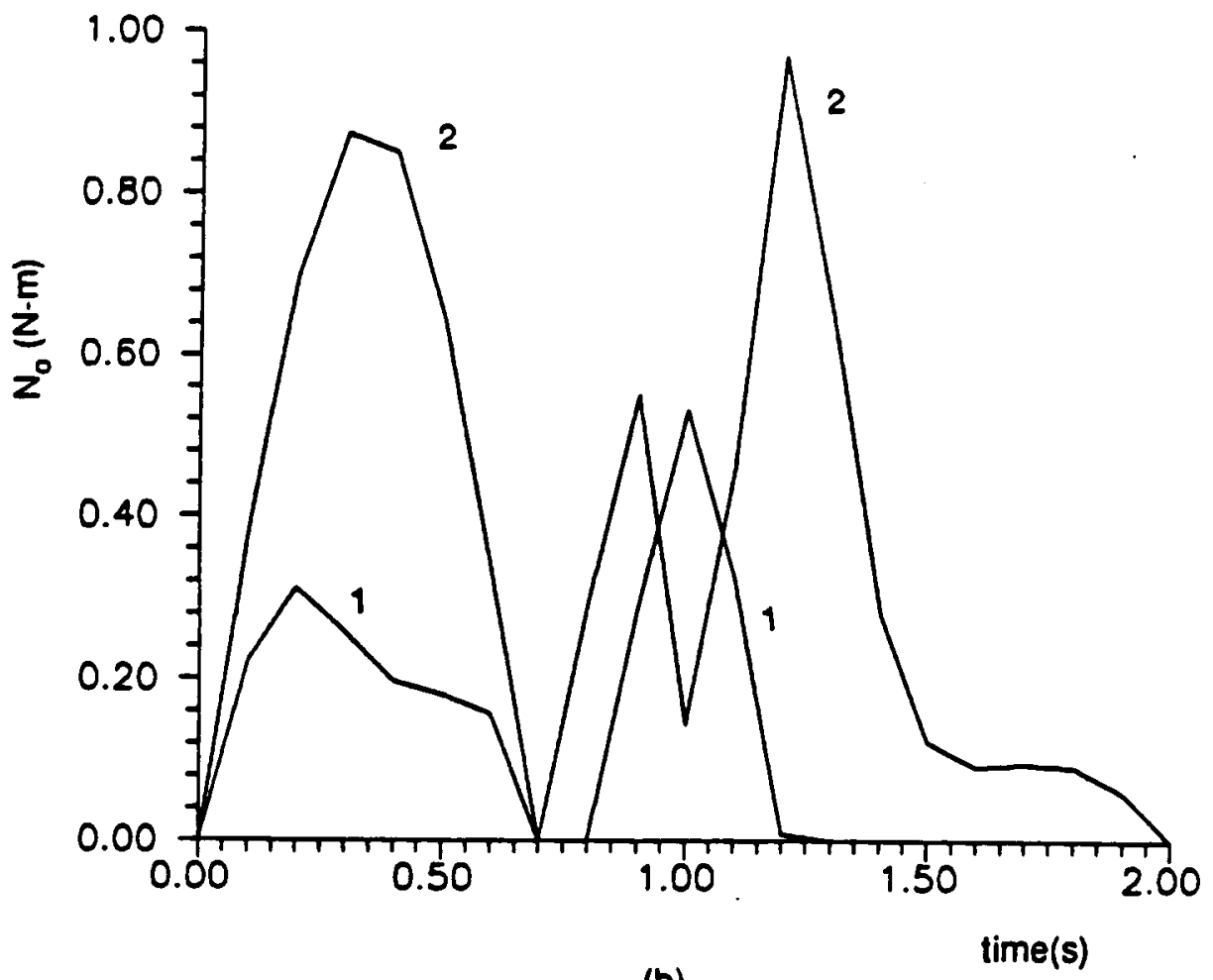

(b)

Fig. 6 Base Reactions Profiles of Case Study (1),(a) Base Force Magnitude and (b) Base Moment Magnitude. 


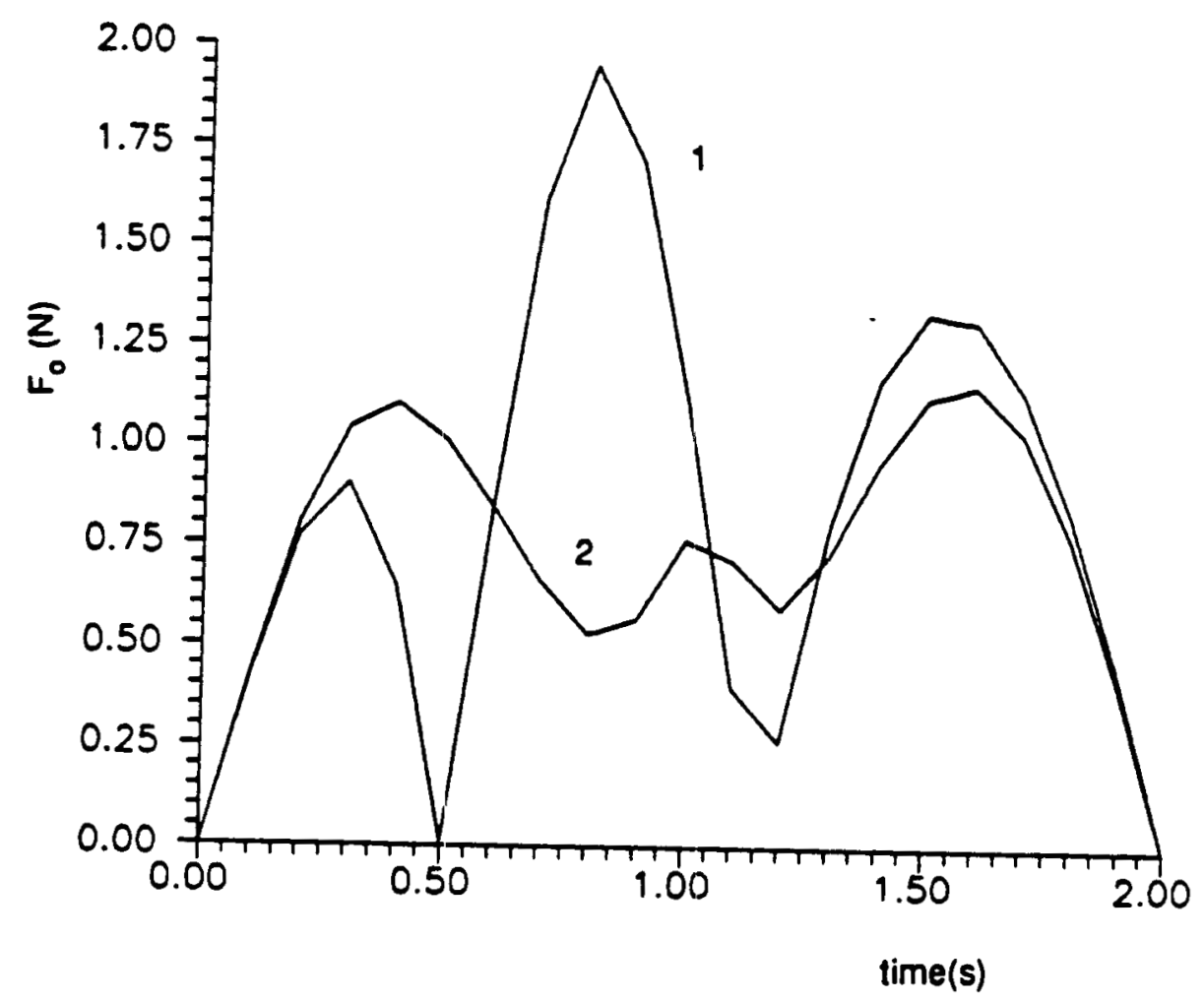

1 - local

$2 \cdot$ global

(a)

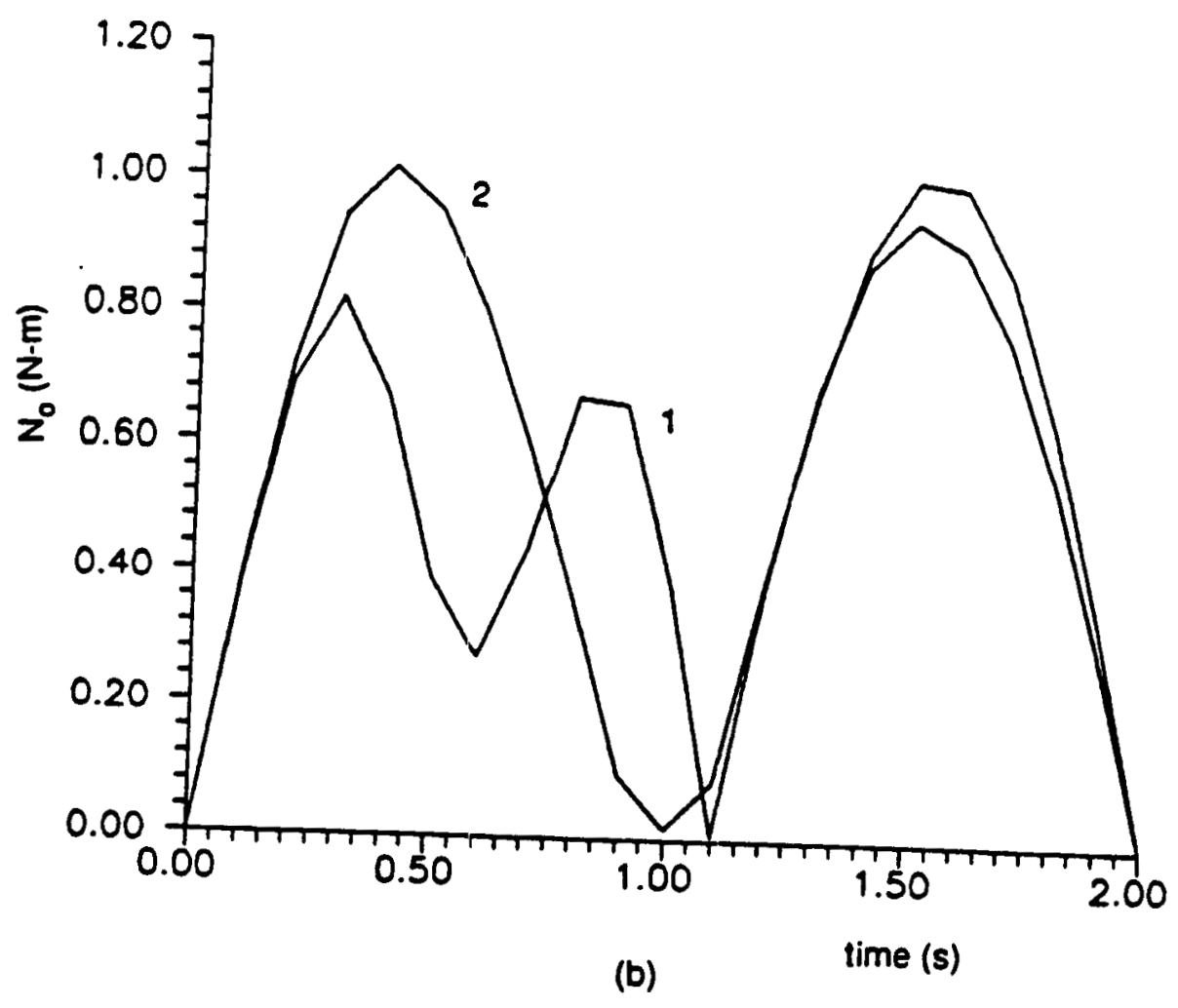

Fig. 7 Base Reactions Profiles of Case Study (2), (a) Base Force Magnitude and (b) Base Moment Magnitude. 


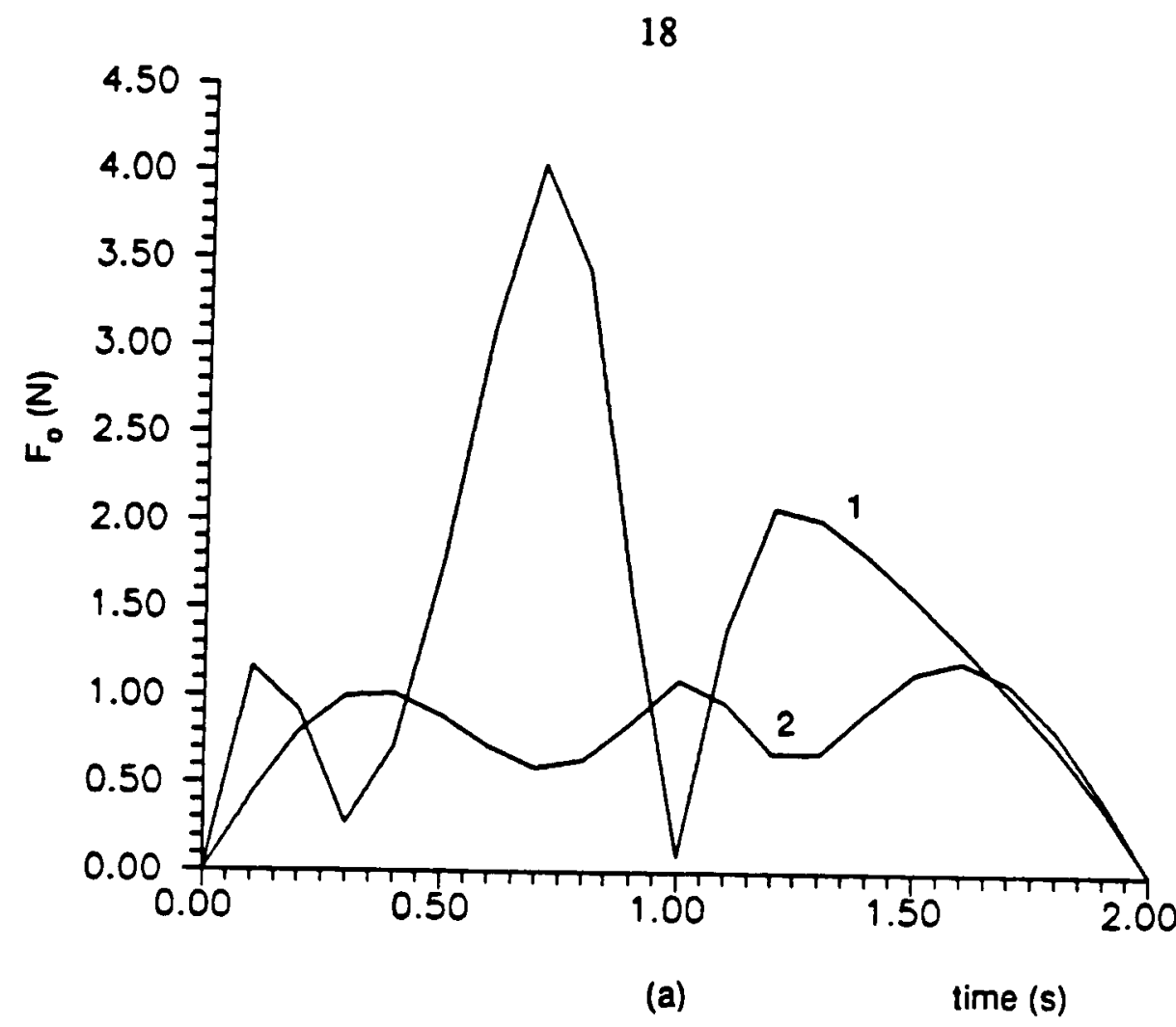

1 - local

2 - global

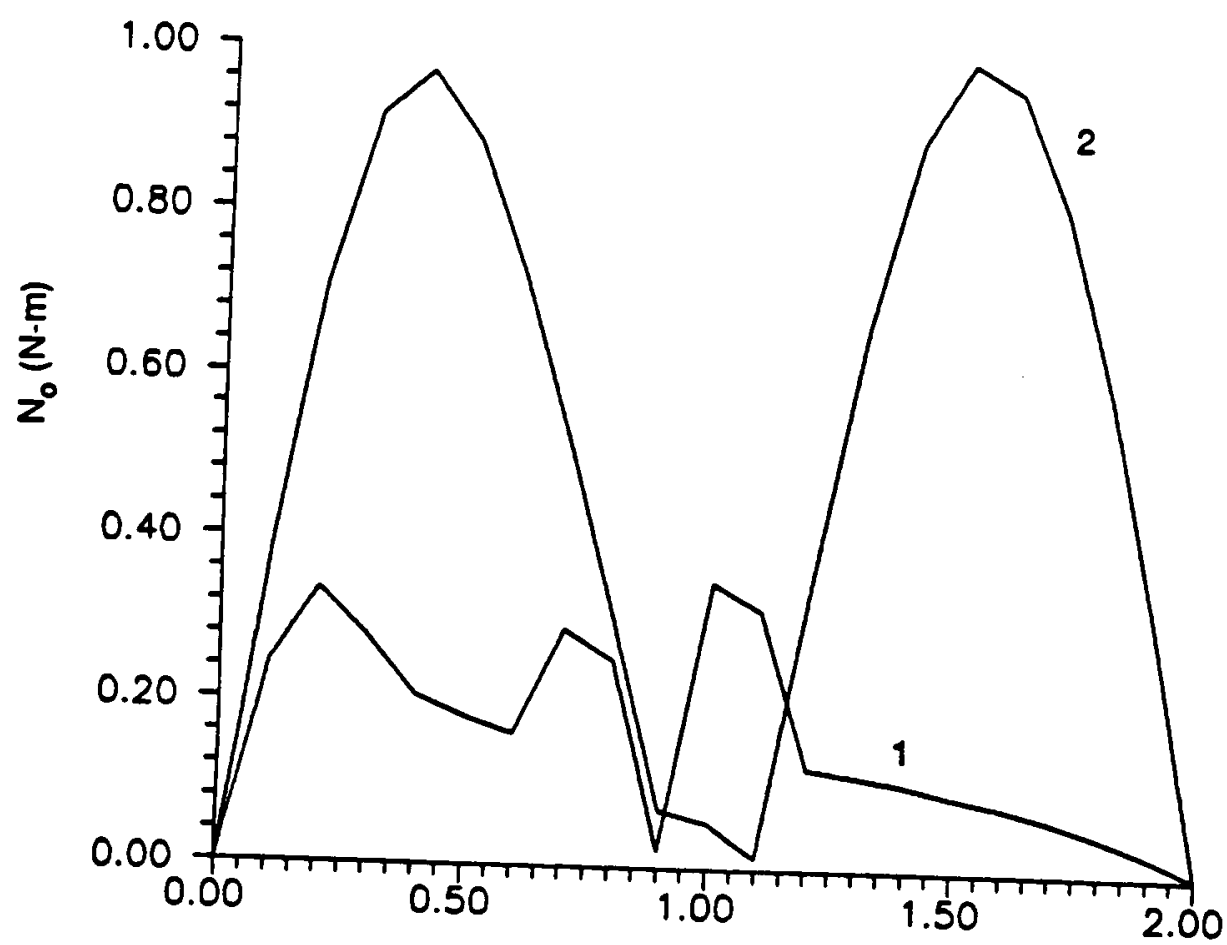

(b)

Fig. 8 Base Reactions Profiles of Case Study (3), (a) Base Force Magnitude and (b) Base Moment Magnitude. 


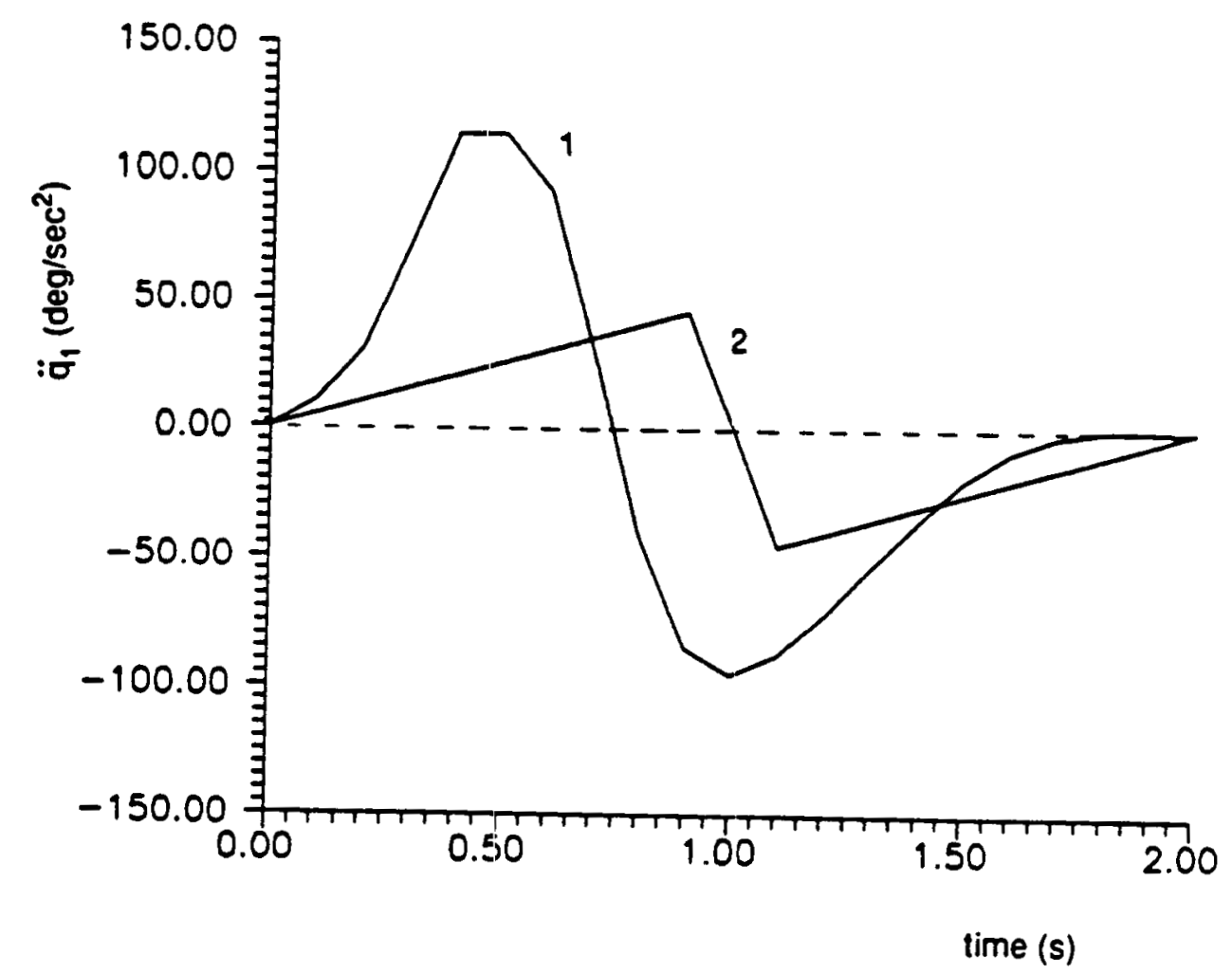

$1 \cdot$ local

$2 \cdot$ global

(a)

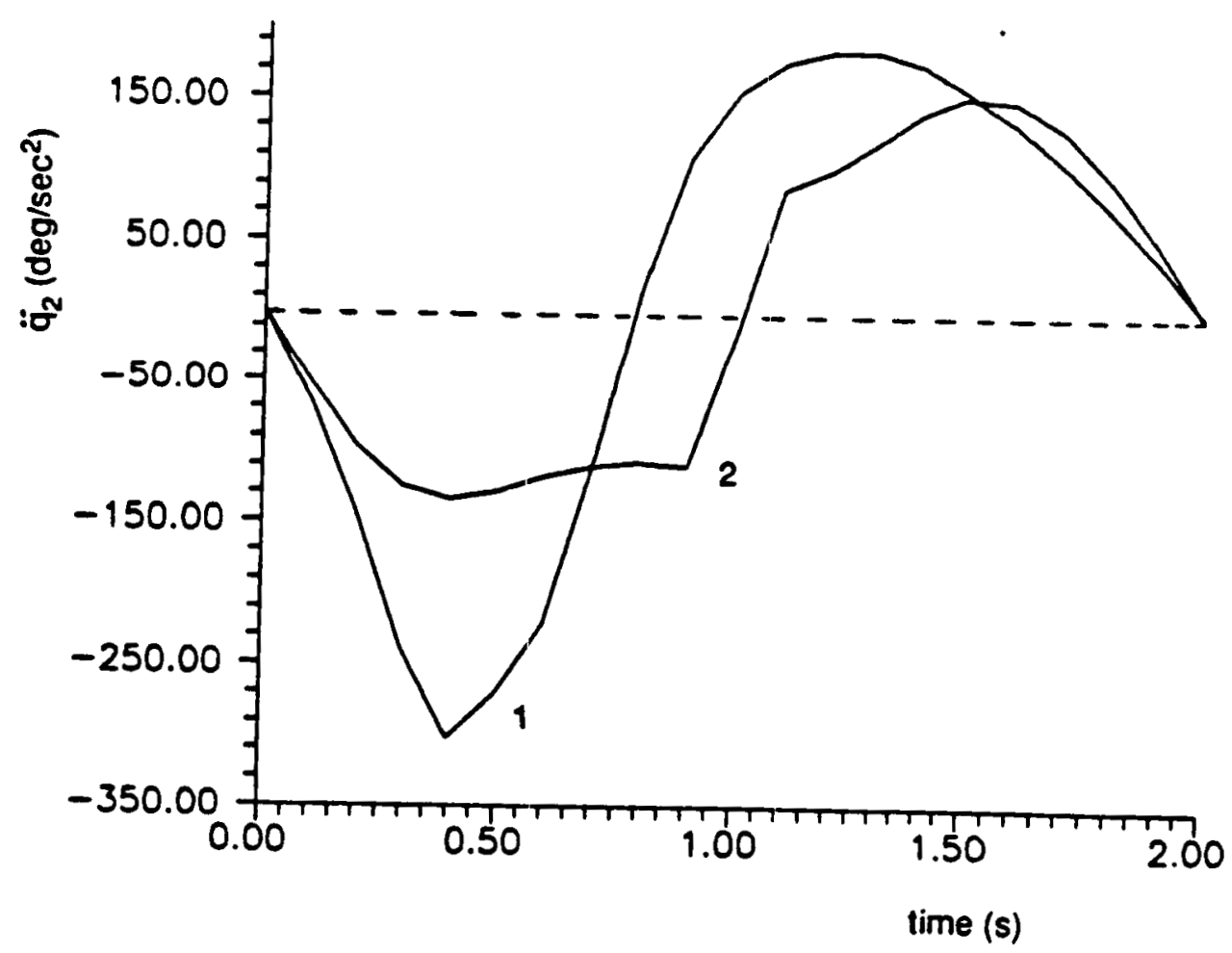

(b)

Fig. 9 Angular Accelerations Profiles, (a) joint 1, (b) joint 2, and (c) joint 3 


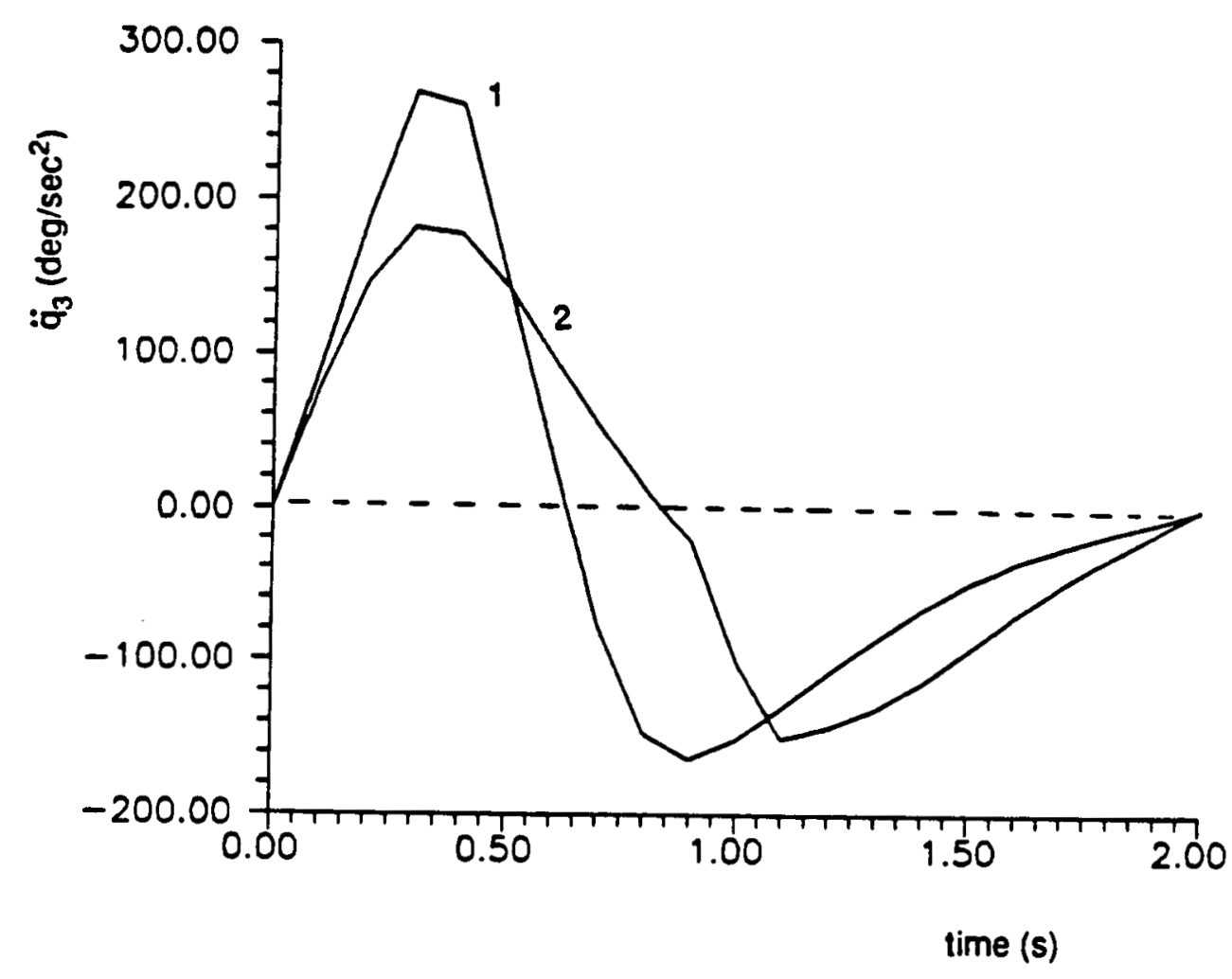

Fig. 9 (c) 


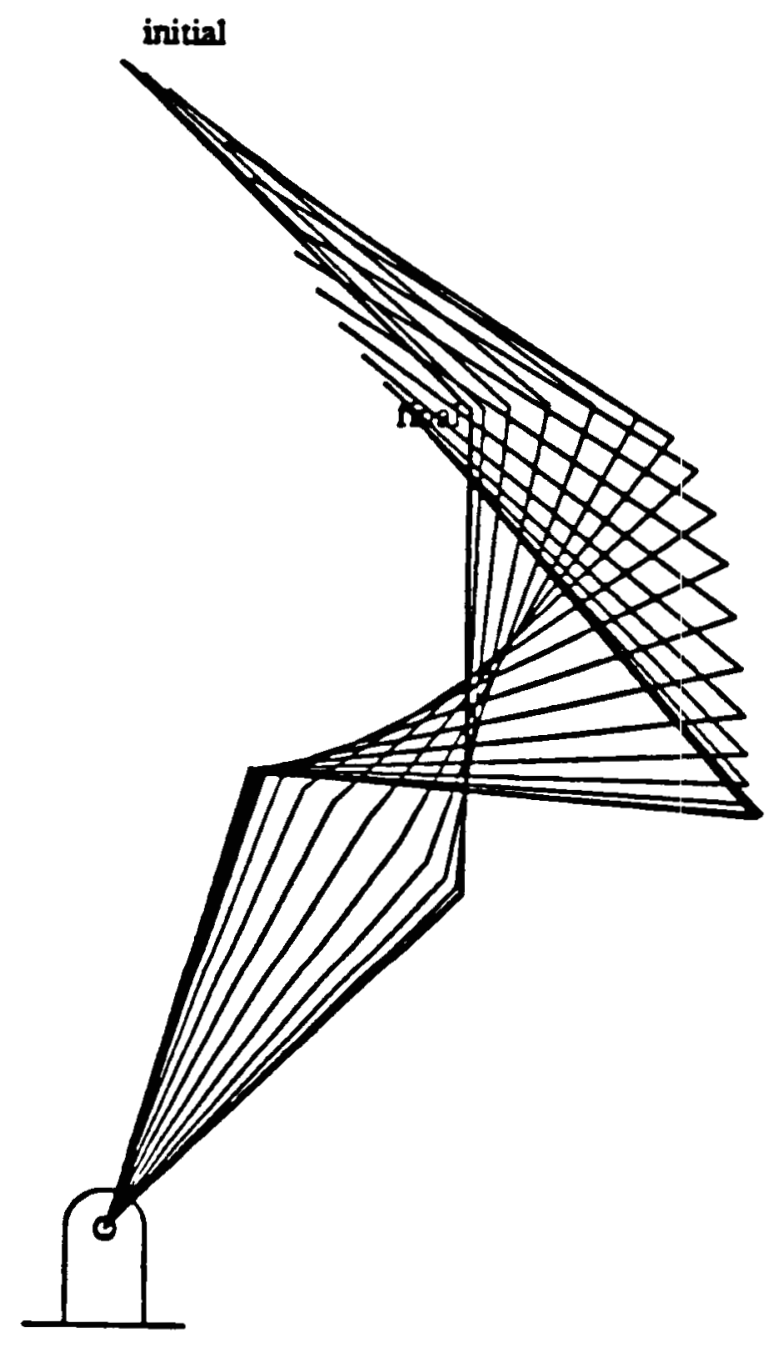

(a) local

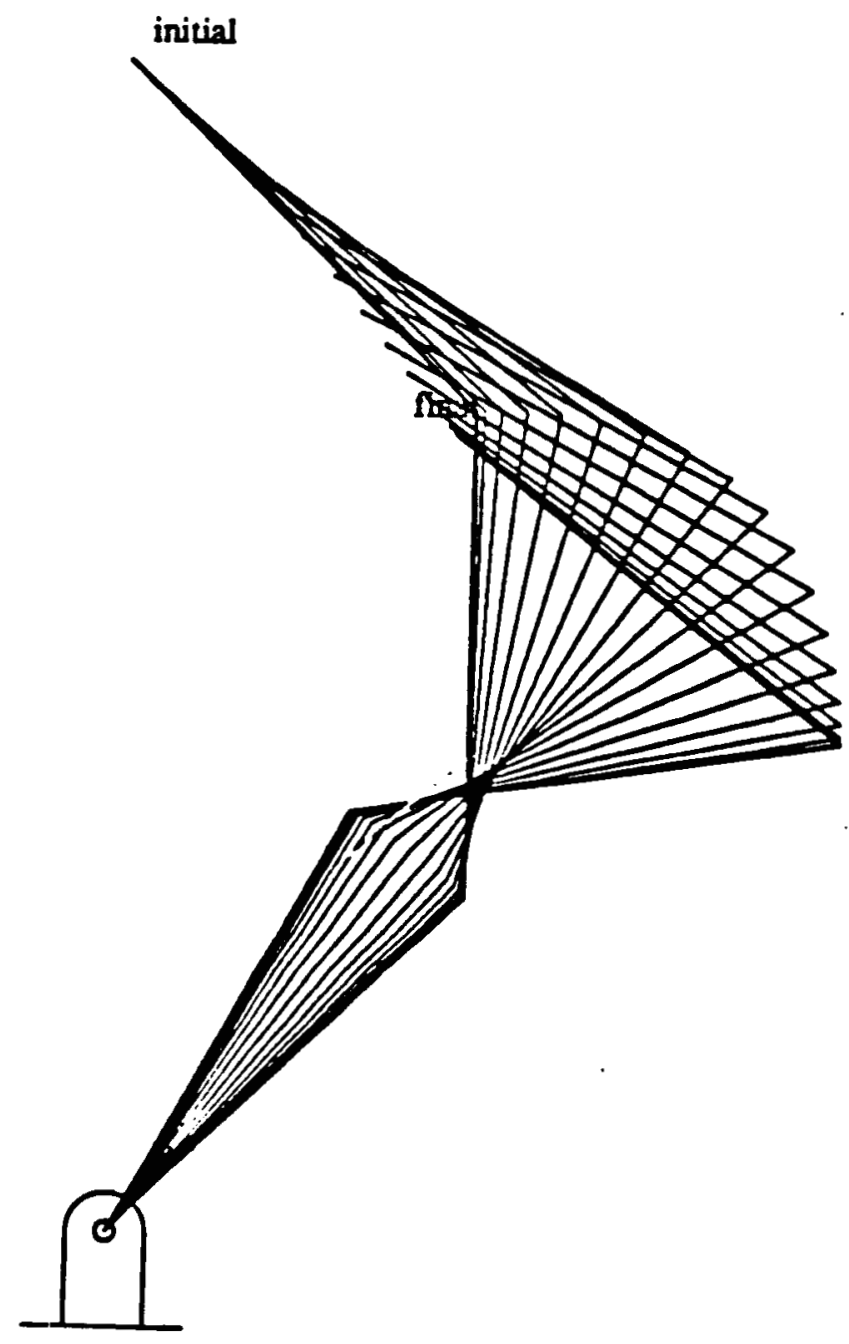

(b) global

Fig. 10 Manipulator Configurations for the Example,(a) local approach and (b) global approach. 


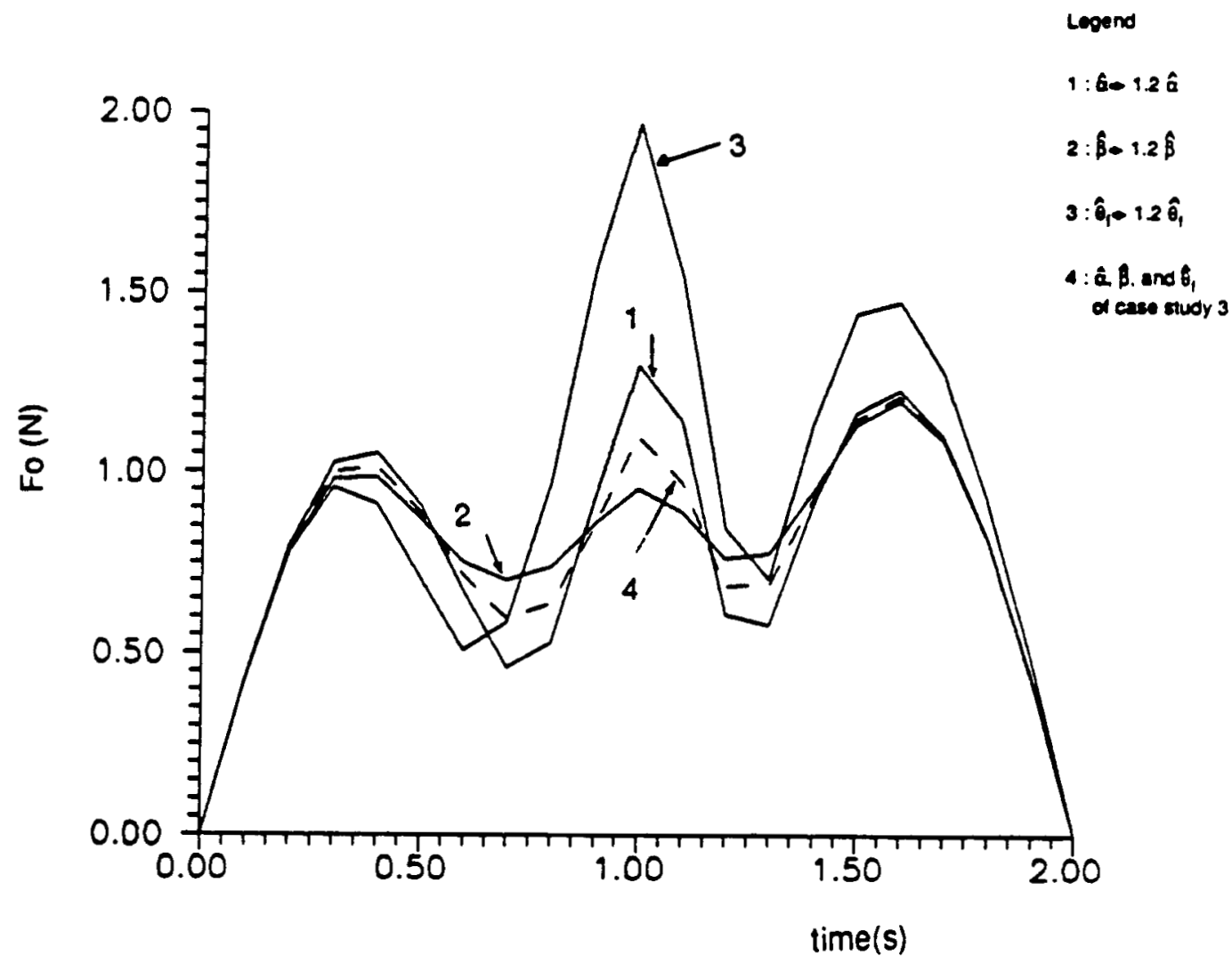

(a)

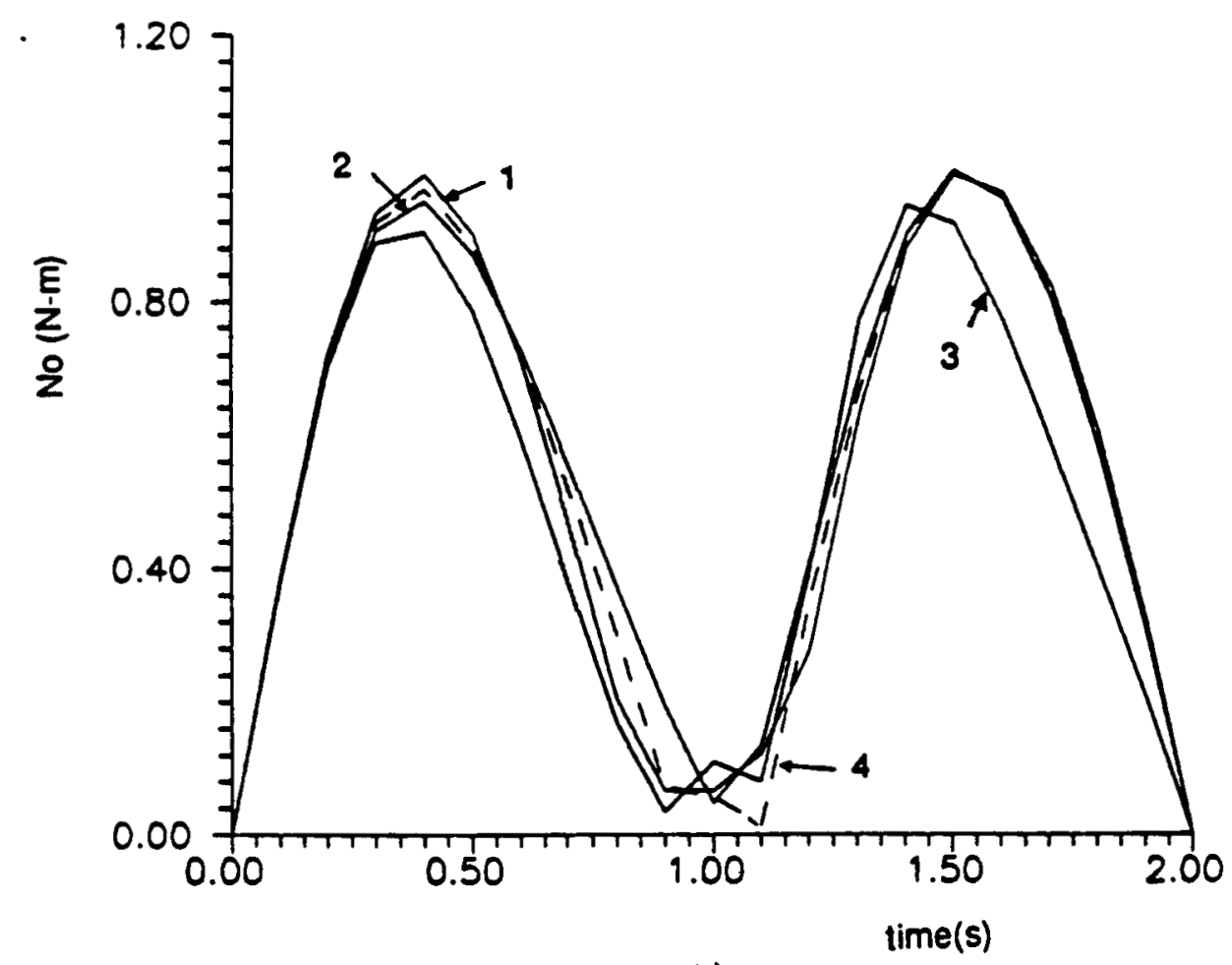

(b)

Fig. 11 Base Reactions Profiles for Case Study 4, (a) Base Force and (b) Base Moment. 
(1)First consider the magnitude of the force transmitted to the base shown in Fig. 6(a) (for Case Study 1), Fig. 7(a) (for Case Study 2), and Fig. 8(a) (for Case Study 3). Each one of these figures has two plots corresponding to the global and local approaches. As might be expected, the magnitude of the base force is the lowest for Case Study 2 where only the base force is being minimized (see Fig. 7(a)). Furthermore, as shown in Fig. 7(a), the global approach "smooths" out the peak in the magnitude of the force which is seen in the local approach at $t=0.8$ second.

(2) Next consider the magnitude of the moment transmitted to the base as shown in Fig. 6(b) (for Case Study 1), Fig. 7(b) (for Case Study 2) and Fig. 8(b) (for Case Study 3). Once again each one of these figures has 2 plots corresponding to the local and global approaches. For the local approach, Case Studies (1) and (3) yield very small base moment, while Case Study (2) (base force minimization only) yields a relatively high base moment. In contrast, for the global approach, the magnitudes of the moment transmitted to the base is virtually independent of the cost function (i.e. whether we are minimizing the magnitudes of the base force, the base moment or the combination of the two). We also see (Figs. 6(b), 7(b), and 8(b)) that the local approach is more effective than the global approach in minimizing the magnitudes of the base moment.

(3) We can summarize the conclusions in (1) and (2) above by the following two statements:

(a) The global approach is the better approach for reducing the magnitude of the base force whereas the local approach is superior for reducing the magnitude of the base moment.

(b) In the global approach it is sufficient to minimize the magnitude of the base force.

(4) The second derivative of the joint: variables (joint "accelerations") for each one of the three joints are shown in Fig. 9, both for the local and global approaches: we observe that very similar acceleration profiles are obtained for these two approaches. The correpsonding manipulator configurations are shown in Fig. 10(a) (for the local approach) and Fig. 10(b) (for the global approach). One should make careful note , both from Figs. 9 and 10 , of the "sense" of motion of the three links. The results shown in Figs. 9 and 10 correspond to Case Study 3.

(5) The results of the simple sensitivity study are shown in Figs. 11(a) (magnitude of the base force) and Fig. 11(b) (magnitude of the base moment) for the global approach and the cost function of Case Study 3. (The optimal results are shown in dashed lines with the perurbed results shown in solid lines). The following two conclusions can be reached from the results as shown in Fig. 11.

1. The magnitude of the base moment is relatively insensitive to small changes in the trajectory (parameters). (Fig. 11 (b)).

2. The magnitude of the base force is quite sensitive to $\theta_{\mathrm{f}}$, the final position of the redundant link (Fig. 11(a)). (The "redundant" link was selected to be the link attached to the base in Fig. 10.)

\section{Summary and Conclusions}

In this paper we have developed a global approach for minimizing the base reactions of a kinematically redundant manipulator used in microgravity environments. The global approach utilizes the Partitioned Jacobian method of redundancy resolution, the 4-3-4 method of joint-trajectory representation and the minimization of a cost function which is a time-integral of the magnitude of the base reactions. We also compared the global approach of this paper with a local approach developed earlier.

The application of the global and local approaches to a planar 3 d.o.f. manipulator with 1 redundant degree of freedom demonstrated the effectiveness of the global approach in smoothing out undesired "peaks" in the force transmitted to the base which were observed in the local approach. We also learned that the global approach was 
more effective in reducing the magnitude of the base force while the local approach was more effective in reducing the magnitude of the base moment. A simple sensitivity analysis performed on the optimal trajectory obtained for the global approach revealed that the magnitude of the force transmitted to the base is more sensitive to the final configuration of the links. We plan to do an extensive sensitivity analysis in the future.

The approach will be implemented on a 4 d.o.f. traction drive manipulator for space application which is currently under construction.

\section{Acknowledgement}

Financial support for this work was provided by the NASA Lewis Research Center under contract NAG3-811.

Appendix 1 : Equations for 4-3-4 joint trajectory representation.

The equations for 4-3-4 joint trajectory representation can be found in [10]. The equations of $\theta_{j}(t), j=1,2,3$ of the three-parameter representation are:

For segment 1,

$$
\begin{aligned}
& \theta_{1}(\tau)=\left(\theta_{f o} \alpha^{\prime}-\sigma\right) \tau^{4}+\sigma \tau^{3}+\theta_{i} \\
& \dot{\theta}_{1}(\tau)=\left[4\left(\theta_{f o} \alpha^{\prime}-\sigma\right) \tau^{3}+3 \sigma \tau^{2}\right] / \tau_{1} \\
& \ddot{\theta}_{1}(\tau)=\left[12\left(\theta_{f o} \alpha^{\prime}-\sigma\right) \tau^{2}+6 \sigma \tau\right] / \tau_{1}{ }^{2}
\end{aligned}
$$

where $\theta_{i}=$ initial joint position,

$$
\begin{aligned}
& \tau=\text { normalized time }(0,1)=t / \tau_{1} \\
& \sigma=f / g \\
& f=2 \theta_{f o} \alpha^{\prime}\left(6+2 \beta^{\prime} / \beta+\beta / \beta^{\prime}\right)-\theta_{f o} \alpha \beta^{\prime} \beta\left(3+\beta^{\prime} / \beta\right)+2 \theta_{f o} \alpha^{\prime} \beta^{\prime} / \beta \\
& g=\beta^{\prime} \beta+3+3 \beta / \beta^{\prime} .
\end{aligned}
$$

For segment 2:

where

$$
\begin{aligned}
& \theta_{2}(\tau)=\left(\theta_{f o} \alpha^{\prime}-v_{1} \tau_{f} \beta-a_{1} \tau_{f}^{2} \beta^{2} / 2\right) \tau^{3}+a_{1} \tau_{f}^{2} \beta^{2} / 2+v_{1} \tau \beta \tau+\theta_{1 f} \\
& \dot{\theta}_{2}(\tau)=\left[3\left(\theta_{f o} \alpha^{\prime}-v_{1} \tau_{f} \beta-a_{1} \tau_{f}^{2} \beta^{2} / 2\right) \tau^{2}+a_{1} \tau_{f}^{2} \beta^{2} \tau+v_{1} \tau \beta\right] / \tau_{2} \\
& \ddot{\theta}_{2}(\tau)=\left[6\left(\theta_{f o} \alpha^{0}-v_{1} \tau_{f} \beta-a_{1} \tau_{f}^{2} \beta^{2} / 2\right) \tau+a_{1} \tau_{f}^{2} \beta^{2}\right] / \tau_{2}^{2}
\end{aligned}
$$

$$
\begin{aligned}
& \tau=\left(t-\tau_{1}\right) / \tau_{2} \\
& v_{1}=\theta_{1}(1) / \tau_{1} \\
& a_{1}=\theta_{1}(1) /\left(\beta^{\prime} \tau_{f}\right)^{2} \\
& \theta_{1 f}=\theta_{1}(1)
\end{aligned}
$$

For segment 3 ,

$$
\begin{aligned}
\theta_{3}(\tau)= & \left(9 \theta_{f o} \alpha^{\prime}-4 v_{2} \beta^{\prime} \tau_{f}-a_{2} \tau_{f}^{2} \beta^{2} R\right) \tau^{4}+\left(-8 \theta_{f o} \alpha^{\prime}+3 v_{2} \beta^{\prime} \tau_{f}\right) \tau^{3}+ \\
& a_{2} \beta^{2} \tau_{f}^{2} \tau^{2} / 2+v_{2} \beta^{\prime} \tau_{f} \tau+\theta_{2 f}
\end{aligned}
$$




$$
\begin{aligned}
& \dot{\theta}_{3}(\tau)=\left[4\left(9 \theta_{f o} \alpha^{\prime}-4 v_{2} \beta^{\prime} \tau_{f}-a_{2} \tau_{f}^{2} \beta^{2} / 2\right) \tau^{3}+3\left(-8 \theta_{f o} \alpha^{\prime}+3 v_{2} \beta^{\prime} \tau_{f}\right) \tau^{2}+\right. \\
& \left.a_{2} \beta^{2} \tau_{f} \tau+v_{2} \beta \tau_{f}\right] / \tau_{3} \\
& \ddot{\theta}_{3}(\tau)=\left[12\left(9 \theta_{f o} \alpha^{\prime}-4 v_{2} \beta^{\prime} \tau_{f}-a_{2} \tau_{f}^{2} \beta^{\prime 2} / 2\right) \tau^{2}+6\left(-8 \theta_{f o} \alpha^{\prime}+3 v_{2} \beta^{\prime} \tau_{f}\right) \tau+\right. \\
& \left.a_{2} \beta^{2} \tau_{f}^{2}\right] / \tau_{3}^{2}
\end{aligned}
$$

where

$$
\begin{aligned}
& \tau=\left(l-\tau_{1}-\tau_{2}\right) / \tau_{3} \\
& v_{2}=3 \theta_{f} \alpha /\left(\tau_{f} \beta\right)-2 v_{1}-a_{1} \tau_{f} \beta / 2 \\
& a_{2}=6 \theta_{f o} \alpha /\left(\tau_{f} \beta\right)^{2}-6 v_{1} /\left(\tau_{f} \beta\right)-2 a_{1} \\
& \theta_{2 f}=\theta_{2}(1) .
\end{aligned}
$$

\section{Appendix 2 : Base Reaction Dynamic Equations}

The following variables are defined

$m_{\mathrm{i}}=$ total mass of the link $i$

$l_{i}=$ length of link $i$

$l_{c i}=$ distance measured from the axis of joint (i-1) to the centroid of link $i$

$I_{\mathrm{i}}=$ moment of inertia about the centroid of link $i$

For the 3 d.o.f. planar manipulator studied in this paper, the base force vector has a $x$-direction component, $\left(F_{\mathrm{o}}\right)_{x}$ and a $y$-direction component, $\left(F_{\partial}\right)_{y}$, and the base moment vector has only a $z$-direction component, $\left(N_{o}\right)_{z}$. The base reactions dynamic equations are given by

$$
F_{b}=M_{b} \ddot{q}+V(q, \dot{q})
$$

where $F_{b}=\left[\left(F_{\alpha}\right)_{x}\left(F_{\partial}\right)_{y}\left(N_{\alpha}\right)_{z}\right]^{T}$.

Let the elements of $M_{b}$ be $m b_{i j},(i=1,2,3)$ and $(j=1,2,3)$. And for convenience, we denote cos(i+...+j) by $c_{i \ldots j}$ and $\sin (i+\ldots+j)$ by $s_{i . . j}$. We have the following equations

$$
\begin{aligned}
& m b_{11}=l_{c 3} m_{3} s_{123}-l_{2} m_{3} s_{12}-l_{c 2} m_{2} s_{12}-l_{1}\left(m_{3}+m_{2}\right) s_{1}-l_{c 1} m_{1} s_{1} \\
& m b_{12}=-l_{c 3} m_{3} s_{123}-l_{2} m_{3} s_{12}-l_{c 2} m_{2} s_{12} \\
& m b_{13}=-l_{c 3} m_{3} c_{123} \\
& m b_{21}=l_{c 3} m_{3} c_{123}+l_{2} m_{3} c_{12}+l_{c 2} m_{2} c_{12}+l_{1}\left(m_{2}+m_{3}\right) c_{1}+l_{c 1} m_{1} c_{1} \\
& m b_{22}=l_{c 3} m_{3} c_{123}+l_{2} m_{3} c_{12}+l_{c 2} m_{2} c_{12} \\
& m b_{23}=l_{c 2} m_{3} c_{123}
\end{aligned}
$$




$$
\begin{gathered}
m b_{31}=l_{1} l_{c 3} m_{3} c_{23}+2 l_{2} l_{c 3} m_{3} c_{3}+2 l_{1} l_{2} m_{3}+2 l_{1} l_{c 2} m_{2} c_{2}+l_{c 3}{ }^{2} m_{3}+ \\
l_{2}{ }^{2} m_{3}+l_{1}{ }^{2} m_{3}+l_{c 2} m_{2} m_{2}+l_{1}{ }^{2} m_{2}+l_{c 1} m_{1}+l_{1}+l_{2}+l_{3} \\
m b_{32}=l_{1} l_{c 3} m_{3} c_{23}+2 l_{2} l_{c 3} m_{3} c_{3}+l_{1} l_{2} m_{3} c_{2}+l_{1} l_{c 2} m_{2} c_{2}+l_{c 3}{ }^{2} m_{3}+l_{2}{ }^{2} m_{3} \\
+l_{c 2}{ }^{2} m_{2}+l_{2}+I_{3} \\
m b_{33}=l_{1} l_{c 3} m_{3} c_{23}+l_{2} l_{c 3} m_{3} c_{3}+l_{c 3}{ }^{2} m_{3} c_{3}+l_{c 3}{ }^{2} m_{3}
\end{gathered}
$$

and the elements of $V(\cdot)$ denoted by $v_{i}$ are

$$
\begin{aligned}
& v_{1}=-\left[\dot{q}_{3}{ }^{2}+2\left(\dot{q}_{2}+\dot{q}_{1}\right) \dot{q}_{3}+\dot{q}_{2}{ }^{2}+2 \dot{q}_{1} q_{2}+\dot{q}_{1}{ }^{2}\right] l_{c 3} m_{3} c_{123}-\left[\left(\dot{q}_{2}{ }^{2}+2 \dot{q}_{1} \dot{q}_{2}+\dot{q}_{1}{ }^{2}\right) l_{2} m_{3}-\right. \\
& \left.\left(\dot{q}_{2}^{2}+2 \dot{q}_{1} \dot{q}_{2}+\dot{q}_{1}^{2}\right) l_{c 2} m_{2}\right] c_{12}-\left(\dot{q}_{1}^{2} l_{1} m_{3}+\dot{q}_{1}{ }^{2} l_{1} m_{2}+\dot{q}_{1}{ }^{2} l_{c 1} m_{1}\right) c_{1} \\
& v_{2}=-\left[\dot{q}_{3}{ }^{2}+\left(2 \dot{q}_{2}+\dot{q}_{1}\right) \dot{q}_{3}+\dot{q}_{2}^{2}+2 \dot{q}_{1} \dot{q}_{2}+\dot{q}_{1}^{2}\right] l_{c 3} m_{3} s_{123}-\left[\left(\dot{q}_{2}{ }^{2}+2 \dot{q}_{1} \dot{q}_{2}+\dot{q}_{1}{ }^{2}\right) l_{2} m_{3}\right. \\
& \left.-\left(\dot{q}_{2}^{2}+2 \dot{q}_{1} \dot{q}_{2}-\dot{q}_{1}^{2}\right) l_{c 3} m_{2}\right] s_{12}-\left[\dot{q}_{1}^{2} l_{1} m_{3}+\dot{q}_{1}^{2}\left(l_{1} m_{2}+l_{c 1} m_{1}\right)\right] s_{1} \\
& v_{3}=-\left[\dot{q}_{3}{ }^{2}+2\left(\dot{q}_{2}+\dot{q}_{1}\right) \dot{q}_{3}+\dot{q}_{2}{ }^{2}+2 \dot{q}_{1} \dot{q}_{2}\right] l_{1} l_{c 3} m_{3} s_{23}-\left(2 \dot{q}_{2} \dot{q}_{3}+2 \dot{q}_{1} \dot{q}_{3}+\dot{q}_{3}{ }^{2}\right) l_{2} l_{c 3} m_{3} s_{3}- \\
& \left(\dot{q}^{2}+2 \dot{q}_{1} \dot{q}_{2}\right) l_{1} l_{2} m_{3} s_{3}-\left(\dot{q}_{2}+2 \dot{q}_{1} \dot{q}_{2}\right) l_{1} l_{c 2} m_{2} s_{2}
\end{aligned}
$$

\section{Appendix 3:Physical Dimensions and Mass Properties}

The physical dimensions and the mass properties of the three degree-of-freedom planar manipulator are given in Table 1. The link length, mass, and inertia of the link are denoted by $l_{i}, m_{i}$, and $I_{i}$ respectively. The centroid of the link is assumed to be at the center of the link.

Table 1 : Simulation Data for 3 d.o.f. Manipulator

\begin{tabular}{cccc}
\hline link & 1 & 2 & 3 \\
\hline$m_{i}(\mathrm{~kg})$ & 1.0 & 1.0 & 1.0 \\
$l_{i}(\mathrm{~m})$ & 0.5 & 0.5 & 0.5 \\
$\mathrm{I}_{i}\left(\mathrm{~kg} \mathrm{\textrm {m } ^ { 2 }}\right)$ & 0.0208 & 0.0208 & 0.0208 \\
\hline
\end{tabular}




\section{References}

1. Lemer, E. J., "Robots on the Space Station," Aerospace America, June 1987, pp. 42-45.

2. Yoshikawa, T., "Analysis and Control of Robot Manipulators with Redundancy," Robotics Research: The First Symposium, ed. M. Brady and R. Paul, Cambridge, Mass., MIT Press.

3. Liegeois, A., "Automatic Supervisory Control of the Configuration and Behavior of Multibody Mechanisms," 1977, IEEE trans. System, Man, Cybernatics, SMC-7, pp. 868-871.

4. Fenton, R. G., Benhabib, B., and Goldenberg, A., "Optimal Point-to-point Motion of Robots with Redundant Degrees of Freedom,"ASME Journal of Engineering for Industry, vol. 108, May 1986, pp.120-126.

5. Hollerbach, J. M., Suh, K. C., "Redundancy Resolution of Manipulators Through Torque Optimization," 1985, IEEE Int. Conf. on Robotics and Automation, St. Louis, Missouri.

6. Suh, K. C., Hollerbach, J. M., "Local Versus Global Optimization of Redundant Manipulators," 1987, IEEE Int. Conf. on Robotics and Automation.

7. Nakamura, Y., Hanafusa, H., "Optimal Redundancy Control of Robot Manipulators," The Int. Journal of Robotics Research, vol. 6 no. 1, spring 87, pp.32-42.

8. de Silva, C. W., Chung, C. L., and Lawrence, C., "Base Reaction Optimization of Robotics Manipulstors for Space Applications," Proc. Int. Symp. Robots, Sydney, Australia, November 1988.

9. Chung, C. L., Desa, S., de Silva, C. W., "Base Reaction Optimization of Redundant Manipulators for Space Applications," Technical Report, of the Robotics Institute, Camegie Mellon University, 1988.

10. Fu, K. S., Gonzalez, R. C., and C. G. S. Lee, Robotics:Control, Vision, and Intelligence, McGraw-Hill, 1987.

11. Asada, H., and Slotine, JJ. E., Robot Analysis and Control, John Wiley and Sons, New York, 1986.

12. Bazaraa, M. S., Nonlinear Programming, John Wiley \& Sons, 1979.

13. Schmitt, D., Soni, A. H., Srinivasan, V', and Naganathan, G., "Optimal Motion Programming of Robot Manipulators," ASME Journal of Mechanisms, Transmissions, and Automation in Design, vol 107, June 1985, pp. 239-244.

14. Ghosal, A., "A New Approach for Kinematic Resolution of Redundancy," Int. Journal of Robotics Research, vol. 7, No. 2, pp. 22-35,1988. 